\title{
Peristaltic Transport of Solid Particles Suspended in a Viscoplastic Fluid: a Numerical Study
}

\author{
N.P. Khabazi, and K. Sadeghy ${ }^{*}$ \\ Center of Excellence in Design and Optimization of Energy Systems (CEDOES), \\ School of Mechanical Engineering, College of Engineering, University of Tehran \\ Tehran, Iran, P.O. Box: 11155-4563, Tel.: (+98) 21 61114011, Fax: (+98) 2188013029 , \\ sadeghy@ut.ac.ir
}

\begin{abstract}
Peristaltic transport of circular rigid particle(s) suspended in a viscoplastic fluid is numerically investigated in a planar two-dimensional channel using the lattice Boltzmann method (LBM) coupled with the smoothed profile method (SPM). Numerical results could be obtained at non-zero Reynolds numbers for a wide range of the propagating wave's parameters. Our numerical results reveal that a fluid's yield stress has a dramatic effect on the peristaltic transport of solid particles. For a single particle suspended in a viscoplastic fluid, which obeys the bi-viscosity (Bingham) model as its constitutive equation, it is found that there exists a threshold wave number below which the yield stress slows down the particle's transport but above which it has an accelerating effect. A fluid's yield stress is also predicted to lower the average velocity of an initially-centered single particle at any given confinement ratio. For a given Bingham number, particle's velocity is predicted to increase by an increase in the confinement ratio provided that it is larger than a critical value. An increase in the Reynolds number may increase or decrease the particle's average velocity depending on the Bingham number. For the two-particle and four-particle scenarios, our numerical results show that the initial positioning of the particles in the channel has a decisive role in their eventual fate.
\end{abstract}

Keywords: Peristaltic flow, yield stress, bi-viscous Bingham model, lattice Boltzmann method, solid particle, smoothed profile method.

\section{Introduction}

A viscous fluid contained in a flexible channel can be forced to flow in a given direction-even at the presence of no favorable pressure gradient-through the generation of a train of contraction/expansion waves propagating along the wall(s) of the channel-the so-called peristaltic flow. Since the pioneering work of Latham [1], this intriguing flow has attracted much attention, thanks primarily to its application in the pump industry for the transport of corrosive and sterile fluids [2-10]. The mechanism is also very attractive for the transport of highly viscous fluids and high-solid slurries. Further interest in this fascinating flow arises from the fact that it is encountered, for example, in human body for the transport of physiological fluids such as urine from the kidney to the bladder, the transport of oxygen-depleted blood from the veins to the heart, and the transport of chyme through our digestive system, among others; see the review by Jaffrin \& Shapiro [8] for a summary of its industrial and physiological instances. In most studies carried out in the past, the suspending fluid medium has been assumed to be a single-phase Newtonian fluid. In industrial and physiological applications, however, one is often faced with the notion that not only the fluid medium is non-Newtonian, but it might also contain large solid particle(s). For example, kidney stone is a common disease nowadays, and so its peristaltic transport through the ureter is a real concern in medical science. Our digestive system should also deal with the transport of solid pills, from time to time, which is a common type of medication. To these should be added the fact that peristaltic pumps are increasingly being considered as the prime mover for the transport of slurries and 
particulate systems. Obviously, having a good knowledge about the parameters which affect peristaltic transport of particles in a non-Newtonian fluid is of great importance in industry and academia, alike.

Hung and Brown [11] appear to be the first ones who experimentally studied the peristaltic motion of a single large particle suspended in a Newtonian fluid. On the theoretical side, early attempts in this area relied on simplifying assumptions such as the Reynolds number being vanishingly small and the wavelength being unduly large [12]. Srivastava and Srivastava [13], and Misra and Pandey [14] resorted to the assumption that the amplitude ratio is very small in order to come up with a semi-analytical solution valid for small particles. Mekheimer et al [15] relied on the long wavelength assumption in their perturbation series to obtain a semianalytical solution for a single particle. A similar idea was utilized by Jimene-Lozano and Sen [16] for a cloud of particles. Although these works have shed some light onto our understanding of peristaltic flow of particulate systems, it should be conceded that due to their approximate nature, the range of their applicability is quite limited. By the advent of more powerful computers and more robust numerical schemes, the need for resorting to such crippling assumptions have been dropped in recent years. One can particularly mention the work carried out by Fauci [17] who employed the immersed boundary method (IBM) for simulating the peristaltic transport of a single solid particle suspended in a Newtonian fluid at high Reynolds numbers. In a more recent work, Connington et al [18] relied on the lattice Boltzmann method (LBM) coupled with the momentum exchange method (ME) in order to tackle the same fluid mechanics problem for Newtonian fluids [18]. The latter work has shown that, for sufficiently large amplitude ratios, the particle ceases to reverse its direction during the particle cycle. More interestingly, a single particle initially placed at the centerline of the channel has been shown to remain at the centerline at all times undergoing no rotation. The case of multiple particles and the interaction between them has not been addressed in any of these works. Also, the above works have ignored the role played by the fluid's rheology on the particle's transport.

To the best of our knowledge, the only work in which the effect of a fluid's rheology has been studied on the peristaltic transport of a single particle is the one carried out by Chrispell and Fauci [19]. Motivated by its physiological implications, they investigated the case in which a neutrally-buoyant particle, placed initially at the channel's centerline, was suspended in a viscoelastic fluid obeying the Oldroyd-B model. They relied on the IBM numerical method for their simulations [19] and showed that the elasticity of the suspending fluid impedes the particle's transport. Although it is true that physiological fluids such as blood, mucus, and synovia are slightly viscoelastic, it should be conceded that under their normal working conditions, their elasticity is of secondary importance as compared with, say, their viscoplastic and/or thixotropic behavior. The same is true for industrial fluids such as waxy crude oils and drilling muds, among others. Surprisingly, however, there appears to be no published work addressing the effects of such important non-Newtonian behavior on the peristaltic transport of solid particles. In the present work, we intend to numerically investigate the effect of a fluid's yield stress on the peristaltic transport of solid particles in a two-dimensional channel at non-zero Reynolds numbers. (The effect of fluid's thixotropy will be addressed in an independent work by us.) To that end, use will be made of the bi-viscous (Bingham) model for representing viscoplastic fluids. We also intend to investigate the effect of different parameters in the peristaltic wave (namely, its wave number and amplitude ratio) on the particle's translational motion and rotation. To achieve these goals, use will be made of numerical methods. But, instead of the IBM scheme used in Ref. 19 or the LBM/ME scheme used in Ref. 18, we have decided to rely on the LBM method combined with the less-demanding smoothed profile method (SPM) [20] for this purpose. The idea of combining these two methods for simulating particulate systems is nothing new and has been beautifully demonstrated in a recent work by Jafari et al [21]. It is, however, the first time that it is being used in simulating peristaltic flow of particulate systems. In practice, the LBM/SPM scheme turned out to be so efficient that it enabled us to obtain converged results, even with a personal computer (PC), not only for the single-particle case but also for the two- and four-particle scenarios.

To reach its objectives, the work is organized as follows: We start with developing the mathematical framework for the peristaltic transport of a viscoplastic fluid obeying the bi-viscous (Bingham) model in a planar flexible channel. We then proceed with describing the numerical method of solution (particularly the SPM) used for 
computing the fluid-particle and particle-particle interactions. Numerical results are presented next addressing the effect of the fluid's yield stress on the flow characteristics over a wide range of wave parameters.

\section{Mathematical Formulation}

We consider peristaltic flow of a viscoplastic fluid containing a rigid two-dimensional circular particle in a planar channel, as shown in Fig. 1. In this figure, $a$ is the half-height of the unperturbed channel, $b$ is the amplitude of the travelling wave, and $c$ is the wave speed. The wavelength of the channel (not shown here) is denoted by $\lambda$.

\section{Figure 1}

The motion of the walls-which is constrained to be in the lateral direction only-is such that a wave is propagating from left to right in the axial direction. The channel is assumed to be infinitely extensible so that it can readily accommodate the prescribed motion. The waves travelling along the upper and lower walls of the channel are assumed to be of the following forms [22]:

$$
\begin{aligned}
& h_{u}(x, t)=+a-b \sin \left[\frac{2 \pi}{\lambda}(x-c t)\right] \\
& h_{l}(x, t)=-a+b \sin \left[\frac{2 \pi}{\lambda}(x-c t)\right]
\end{aligned}
$$

where subscripts " $u$ " and "l" refer to the upper and lower walls, respectively. The channel is assumed to comprises an infinite (integer) number of such propagating waves. To be able to validate our code with published data, which is available for peristaltic transport of a single neutrally-buoyant particle suspended in a Newtonian fluid $[17,18]$, just one wavelength is used as our computational domain. With an eye on the physiological applications of our work, we only consider the case of "free-pumping". That is to say that, it is assumed that the flow is not assisted by an externally-imposed favorable pressure gradient. To further simplify the analysis, we assume that the effects of gravitational force are negligible, i.e., the particle and the fluid have the same density. We also assume that the fluid and the particle are initially at rest- by default, the particle(s) is assumed to be initially located at the centerline of the channel unless otherwise is stated. The flow is initiated at time $\mathrm{t}=0$ as soon as the flexible walls of the channel start to oscillate vertically, as described by Eqs. 1 and 2 . The equations governing the fluid flow are then the Cauchy equations of motion together with the continuity equation [23]:

$$
\begin{aligned}
& \rho_{f}\left(\frac{\partial \mathbf{u}}{\partial t}+\mathbf{u} \cdot \nabla \mathbf{u}\right)=-\nabla p+\nabla \cdot \underset{\sim}{\tau}, \\
& \frac{\partial \rho_{f}}{\partial t}+\nabla \cdot\left(\rho_{f} \mathbf{u}\right)=0,
\end{aligned}
$$

where $\mathbf{u}$ is the velocity vector, $\rho_{f}$ is the fluid's density, $p$ is the isotropic pressure, and $\tau$ is the extra stress tensor. As to the boundary conditions needed to close the problem, we rely on the no-slip and no-penetration conditions at the walls. Like Refs 17-19, we resort to periodic boundary conditions at the two ends of the domain or our simulations. As to the stress tensor, we assume that the fluid of interest obeys the bi-viscosity (Bingham) model, as proposed originally by Beverly and Tanner [24]. In our recent work [22] we have explained in some details why this model is advantageous over the classic Bingham model for capturing the shape of the plug(s) formed in peristaltic flows of homogenous viscoplastic fluids. In short, it can be said that unlike the classic Bingham model, in the bi-viscosity model the un-yielded region is treated as a highly-viscous 
fluid in which small deformation is allowed to occur. And, as soon as the material starts to yield, it flows with a much lower viscosity in the yielded region. As a result of this little maneuvering, the state of stress becomes determinable in the whole domain (even in the un-yielded region) so that the shape of the yield surface can easily be computed using velocity-based viscous solvers such as LBM/SPM. The constitutive equation for this useful viscoplastic fluid model reads as [24]:

$$
\tau_{i j}= \begin{cases}2\left(\mu_{p}+\frac{\tau_{y}}{\dot{\gamma}}\right) d_{i j} & ; \quad \text { for } \dot{\gamma} \geq \dot{\gamma}_{c} \\ 2 \mu_{0} d_{i j} & ; \quad \text { for } \dot{\gamma}<\dot{\gamma}_{c}\end{cases}
$$

where $\mu_{0}$ is the large viscosity of the un-yielded region, $\mu_{p}$ is the small viscosity of the yielded zone, $\dot{\gamma}$ is the second invariant of the rate-of-deformation tensor $\left(d_{i j}\right)$, and $\dot{\gamma}_{c}=\tau_{y} /\left(\mu_{0}-\mu_{p}\right)$ is the critical shear rate, i.e., the shear rate at which the flow curve suddenly changes its slope.

\section{Numerical Method}

In the present work, like our recent publication [22], we have decided to rely on the lattice Boltzmann method (LBM) for solving the equations of motion. To take care of the interaction between the fluid and the particle(s) and also between the particles themselves, we had at our disposal the immersed boundary method used by Fauci [18], and the momentum exchange method used by Connington [18]. The latter method was used with no major difficulty for simulating the interaction between the flexible walls of the channel and the fluid. We were faced with numerical difficulties when using either of these two methods for simulating close-range particle-particle interactions even for Newtonian fluids. As the last resort, we tried the smoothed profile method (SPM) for this purpose and reached to the conclusion that it would not suffer from numerical instabilities even for viscoplastic fluids. The SPM was originally developed by Nakayama and Yamamoto for simulating the sedimentation of particles in colloidal systems [20]. In a subsequent work, it was coupled with the LBM for simulating such flows with great success [21]. The idea was to first use the lattice Boltzmann equation for solving the equations of motion in the entire domain (i.e., in the fluid region as well as the in the solid regions). The effect of the particle could then be introduced through a body force term which was added to the lattice Boltzmann equation through the SPM scheme. To the best of our knowledge, this idea has not previously been implemented in any peristaltic flow study involving particles. In our recent work [22] we have described in some details the lattice Boltzmann method (LBM) used for simulating peristaltic flow of a single-phase viscoplastic fluid obeying the bi-viscous model. The same code was modified to incorporate the smoothed profile method for the present work. For this reason, we only focus on describing the latter method in some details here.

\subsection{Smoothed Particle Method (SPM)}

Technically-speaking, in our numerical scheme the role of the smoothed profile method (SPM) is to distinguish between solid region(s) and the fluid region. It is also used to enforce the no-slip condition at the interface. To that end, the surface of the particle is treated as an interface having a width comparable to the grid spacing. A density profile function, $\varphi_{i}(\mathbf{x}, t)$, is then introduced which is set equal to "zero" in the fluid region and to "one" in the main body of the particle. A continuous profile is then used to ensure a smooth transition from "zero" to "one" across the width of the interface (see Fig. 2).

Figure 2

For a fluid system comprising $\mathrm{N}$ circular particles (which is, at most, equal to 4 in our peristaltic simulations) the above-mentioned density function can be obtained through a summation over all particles; that is [20]: 
$\varphi(\mathbf{x}, t)=\sum_{i=1}^{N} \varphi_{i}(\mathbf{x}, t)$

where $\varphi_{i}$ is the density profile for the $i$-th particle. In practice, the density profile lies in the range of $0 \leq \varphi_{i} \leq 1$ with zero denoting the fluid region and one denoting the solid region(s). Across the finite-thickness of the interface, it must smoothly vary from zero to one through the use of an appropriate function, $\mathrm{s}(\mathbf{x}, \mathrm{t})$; that is:

$\varphi_{i}(\mathbf{x}, t)=s\left(R_{i}-\left|\mathbf{x}-\mathbf{R}_{i}(t)\right|\right)$

where $R_{i}$ is the radius of the $i$-th particle with $\boldsymbol{R}_{i}$ being the position vector of its centre-of-mass. As to the sfunction, the following function, as proposed in Ref. 20 , has been found to be appropriate for our simulations:

$s(x)= \begin{cases}0 & ; \text { for } x<-\delta / 2 \\ \frac{1}{2}\left[\sin \left(\frac{\pi x}{\delta}\right)+1\right], & ; \text { for }|x| \leq+\delta / 2 \\ 1 & ; \text { for } x>+\delta / 2\end{cases}$

where $\delta$ is the finite thickness of the interface. At any given time, $t$, the velocity field of all points (nodes) on the particle (i.e., $\varphi \mathbf{u}_{p}$ ) can be obtained as:

$\boldsymbol{\varphi}\left(\mathbf{x}, t_{n}\right) u_{p}\left(\mathbf{x}, t_{n}\right)=\sum_{i=1}^{N} s\left(R-\left|\mathbf{x}-\mathbf{R}_{i}\left(t_{n}\right)\right|\right) \times\left[\mathbf{U}_{c, i}\left(t_{n}\right)+\boldsymbol{\omega}_{i}\left(t_{n}\right) \times\left\{\mathbf{x}-\mathbf{R}_{i}\left(t_{n}\right)\right\}\right]$

where $\mathbf{U}_{c, i}$ is the translational velocity of the center-of-mass of the $i$-th particle with $\Omega_{i}$ being its angular velocity. Knowing the initial velocity and position of the particle(s), we can proceed with calculating their values at an updated time. To that end, it is assumed that the fluid nodes covered by the particle has the same velocity as the particle itself. To force the fictitious fluid to satisfy the rigid-body motion constraint, a body force is then introduced over the particle inner nodes-obviously, this force must be set equal to zero outside the solid domain. Denoting this force by $\mathbf{f}_{H}(\mathbf{x}, t)$ we have:

$\mathbf{f}_{H}(\mathbf{x}, t)=\rho_{f} \varphi(\mathbf{x}, t)\left[\mathbf{u}_{P}(\mathbf{x}, t)-\mathbf{u}(\mathbf{x}, t)\right]$,

where $\mathbf{u}(\mathbf{x}, t)$ refers to the fluid's velocity. (The interaction force, $\mathbf{f}_{H}(\mathbf{x}, t)$, is a result of a difference between the particle's velocity and the fluid's velocity at any given point used in the domain to enforce the no-slip condition at the fluid/particle interface.) A force equal to, but opposite, this force is exerted on the fluid. This is the force which must be inserted into the collision function in the LBM method-this is how the two methods are coupled To update the translational and angular velocities of the particles, we rely on the momentum equation. By integration over all of the particles, the (drag) force and the torque exerted on the particle (say, by the fluid surrounding the particle) can be obtained as,

$$
\begin{aligned}
& \mathbf{F}_{i}^{H}=\int_{\forall_{i}} \rho_{f} \varphi_{i}(\mathbf{x}, t)\left[\mathbf{u}(\mathbf{x}, t)-\mathbf{u}_{P}(\mathbf{x}, t)\right] d \forall, \\
& \mathbf{T}_{i}^{H}=\int_{\forall_{p_{i}}}\left(\mathbf{x}-\mathbf{R}_{i}^{n}\right) \times \rho_{f} \varphi_{i}(\mathbf{x}, t)\left[\mathbf{u}(\mathbf{x}, t)-\mathbf{u}_{P}(\mathbf{x}, t)\right] d \forall .
\end{aligned}
$$

where $\forall_{i}$ stands for the volume of the particle and the superscript "H" refers to the host fluid. Since the particles' motion and rotation are governed by the Newton/Euler equations, we have [20]: 
$M_{i} \frac{d \mathbf{U}_{c_{i}}}{d t}=\mathbf{F}_{i}^{H}+\mathbf{F}_{i}^{p p}+\mathbf{F}_{i}^{p w}+\mathbf{F}_{i}^{B}$,

and,

$I_{i} \frac{d \boldsymbol{\omega}_{i}}{d t}=\mathbf{T}_{i}^{H}$,

where $M$ is the mass of the $i$-th particle, $I_{i}$ is the moment-of-inertia tensor of the $i$-th particle, $\boldsymbol{\Omega}_{i}$ is its angular velocity vector, $\mathbf{F}_{i}^{B}$ is the buoyancy force (which is zero for suspending particles), $\mathbf{F}_{i}^{p p}$ is the particle-particle collisional repulsive force, and $\mathbf{F}_{i}^{p w}$ is the particle-wall external repulsive force. These latter two (repulsive) forces are included in order to ensure that particles do not penetrate into each other or through the channel's walls. In practice, they are determined using theoretical formulations derived by Glowinski et al [25], and Feng and Michaelides [26], respectively. The particles' angular velocity can then be updated as:

$\boldsymbol{\omega}_{i}^{n+1}=\boldsymbol{\omega}_{i}^{n}+\boldsymbol{I}_{i}^{-1} \int_{t}^{t+\Delta t} \mathbf{T}_{i}^{H} d s$.

where $\mathbf{I}_{\mathrm{i}}$ is the moment of inertia tensor for the $i$-th particle. Knowing the updated angular velocity, the updated velocity field for the particle's inner nodes can easily be obtained. To that end, we first update the translational velocity of the particle's center-of-mass; that is:

$\mathbf{u}_{c, i}^{n+1}=\mathbf{u}_{c, i}^{n}+M_{i}^{-1} \int_{t_{n}}^{t_{n}+\Delta t} \mathbf{F}_{i}^{H}+\mathbf{F}_{i}^{p p}+\mathbf{F}_{i}^{p w}+\mathbf{F}_{i}^{B} d s$,

We then proceed with updating the $\mathbf{u}_{p}$ as:

$\mathbf{u}_{p}^{n+1}=\mathbf{u}_{c_{i}}^{n+1}(t)+\boldsymbol{\omega}_{i}^{n+1}(t) \times\left[\mathbf{x}-\mathbf{R}_{i}^{n+1}(t)\right]$

where the new position of the particle is calculated by:

$$
\mathbf{R}_{i}^{n+1}=\mathbf{R}_{i}^{n}+\int_{t_{n}}^{t_{n}+\Delta t} \mathbf{u}_{c, i} d s .
$$

The updates velocity and positions so-obtained are then used to calculated the density and velocity fields for the next time step. The above formulations were translated into a FORTRAN code which was run on a Pentium 4 personal computer (PC). Depending on the set of parameters chosen for the simulations, each run took us between nearly one hour to a couple of days to converge. As convergence criterion, we have monitored the average axial velocity $(\bar{U})$, calculated over two consecutive particle cycles, to see when its variation is less than one percent. The time-averaged axial velocity is defined as:

$\bar{U}=\frac{1}{T_{p}} \int_{t}^{t+T_{p}} U d s$,

where $T_{p}$ is the period of the particle (which is, in general, different from the period of the peristaltic wave), and $U=u_{p} / c$ is the instantaneous axial velocity-made dimensionless through dividing by $\mathrm{c}$. The other dimensionless parameters involved in our fluid mechanics problem are the Reynolds number (Re), the Bingham number $(\mathrm{Bn})$, the wave number $(\alpha)$, the confinement ratio $(\beta)$, the amplitude ratio $(\phi)$, the density ratio $(\sigma)$, and the dimensionless time $(\Lambda)$. They are defined, respectively, as:

$$
R e=\frac{\rho_{f} c a}{\mu_{p}} \alpha ; B n=\frac{\tau_{y} a}{c \mu_{p}} ; \alpha=\frac{a}{\lambda} ; \beta=\frac{R}{a} ; \varphi=\frac{b}{a} ; \sigma=\frac{\rho_{s}}{\rho_{f}} ; \Lambda=\frac{c t}{\lambda}
$$


where $R$ is the radius of the particle, and $\rho_{\mathrm{s}}$ is the particle's density. (It is worth-mentioning that in Ref. 18 the confinement ratio, $\beta=R / a$, has been labeled as the volume fraction.) In all simulations carried out in this work, we have assumed that the density ratio is equal to one (i.e., $\sigma=1$ ) - this is tantamount to saying that buoyancy force is not involved in our problem.

\subsection{Code Verification}

The LBM/SPM code developed in this work could easily reproduce LBM/IBM results reported by Feng and Michaelides [26] for the sedimentation of two particles aligned at a distance (in the vertical direction) in a vessel filled with a Newtonian fluid-see Ref. 27 for the comparison. As a more stringent test, we have decided to check the performance of the code in its dealing with the task of simulating the peristaltic motion of a single particle suspended in a Newtonian fluid. This test case has been studied in some details by Fauci [17] using the IBM method, and by Connington et al [18] using the LBM/ME method. Figure 3 shows a comparison between our LBM/SPM method with their results. This figure shows that, as far as the axial location and axial velocity of the particle are concerned, our code is doing a nice job in this challenging fluid mechanics problem. It is worthmentioning that, the results reported in Fig. 3 have been obtained when one wavelength is used as the computational domain. Connington et al [18] have also investigated the effect of the domain's size (in terms of the number of wavelengths) on the numerical results. Figure 4 shows a comparison between our numerical results with their results when several wavelengths are used as the domain-all containing just one particle. This figure shows that our code is well capable of reproducing the results reported in Ref. 18, and this is particularly so at small confinement ratios. In fact, for a domain comprising just one wavelength, the difference between the two sets of results is negligible even for $\beta$ as large as 0.65 , where obviously we should have $\beta \leq 1-\varphi$ for the particle to pass through the minimum area.

\section{Figure 3}

\section{Figure 4}

In addition to serving as a challenging test to verify our code with published data, Fig. 4 also reveals the importance of the domain's length on the numerical results. That is to say that, as originally noted by Connington et al [18], this figure clearly shows that the analysis of one wavelength is not identical to the analysis of an integer number of the wavelength, when only one particle is involved (see Fig. 5). This is not surprising anymore [see Ref. 18] when it is realized that for a simulation in which only one wavelength is used as the computational domain, the particle is just one wavelength away from its periodic copy. Thus, there is a strong interaction between the particle and its periodic copy. This interaction is weakened when the size of the domain is increased because now the distance between the particle and its periodic copy is increased (see Fig. 5). That is to say that, we will be dealing with a different fluid mechanics problem each time we increase the size of the domain. In a sense, the single wavelength domain is more attractive in that it better represents dense particulate systems. This is perhaps why Connington et al [18] proceeded with just one wavelength as the domain in the rest of their work even after discovering the importance of the domain's length. With the same token, and also to be able to validate our code with their code, we are going to report results for just one wavelength as the computational domain. (We regard, our work as an extension of the work carried out by Connington et al [18] for Newtonian fluids.)

Figure 5

To ensure that the results are mesh-independent we have tried three different mesh sizes of $100 \times 60,200 \times 120$ and $400 \times 240$ lattice units (where the first number means lattice units in the $\mathrm{x}$-direction with the second number referring to the lattice units in the y-direction) in order to check the spatial convergence of the method. As can be seen in Fig. 6, the results obtained using the latter two grids are virtually the same. To reduce the 
computational time, we have decided to rely on $200 \times 120=24,000$ grids for our simulations in the rest of the work.

\section{Figure 6}

An important issue to be addressed is the order of the spatial convergence for our LBM/SPM code. It is well established that in homogenous flows, the LBM alone has an order of spatial convergence between one and two-depending on the boundary conditions [28]. To see if it is also true when LBM is coupled with the SPM, we need a test case for which an analytical solution does exist, and the circular Couette flow of Newtonian fluids appears to be a good candidate for this purpose. For the case in which the inner cylinder is rotating with the outer cylinder being stationary, the tangential velocity profile can be found in any reference textbook as [23]:

$u_{\theta}(r)=\Omega\left[\frac{\left(R_{1} R_{2}\right)^{2}}{\left(R_{2}^{2}-R_{1}^{2}\right)}\left(\frac{1}{r}-\frac{r}{R_{2}^{2}}\right)\right]$

where $R_{1}$ and $R_{2}$ are the inner and outer radii, and $\Omega$ is the angular velocity of the inner cylinder. Our LBM section of the code could easily recover this velocity profile while the SPM section of the code treats the inner cylinder as a rigid particle [27]. By changing the lattice size, for a given Reynolds number, the error incurred in computing the true tangential velocity can be estimated using the following equation [28],

Error $=\sqrt{\frac{\sum_{i=1}^{n}\left(u_{\theta, c}-u_{\theta}\right)^{2} / u_{\theta}^{2}}{n}}$.

where $u_{\theta, c}$ is the computed velocity, and $n$ is the number of points over which the computation is carried out.

Figure 7a shows the error calculated this way versus the lattice spacing in a log-log plot at two different Reynolds numbers. The results clearly show that the spatial convergence of our LBM/SPM code is roughly one for circular Couette flow of Newtonian fluids. Unfortunately, there is no analytical solution available for peristaltic flow of particulate systems, even for Newtonian fluids. Still, to get an idea as to how the geometry affects the incurred error, we have obtained numerical results for the average velocity of a single particle nine different grid sizes with the finest being $400 \times 520$. Figure $7 \mathrm{~b}$ shows the error calculated using Eq. 21 for a typical $R e=5, \alpha=0.5, \beta=0.2, \varphi=0.25$, and $B n=5$. This figure shows that the grid size indeed affects the error. This figure also shows that, in comparison with the first test case, the data are more scattered for the second test case (i.e., the peristaltic case). To explain the scatter, it should be noted that unlike circular Couette flow, peristaltic flow is an unsteady flow by nature. To check if convergence has been achieved for the peristaltic case we had to compute the (average) axial velocity of the particle over two consecutive particle cycles and see if the difference is less a certain criterion. In the present work, we have set the convergence criterion at $1 \%$, as suggested in Ref. 18 . We have reached to the conclusion that this rather rough criterion is mainly responsible for the scatter seen in Fig. 7 b. Indeed, by trying a stricter criterion of $0.1 \%$ instead of $1 \%$ (just for a couple of data points) we have found out that the scatter is significantly decreased with the price being that the computational time is dramatically increased. Interestingly, however, the slope of the regression line remains virtually intact even when a stricter criterion is used, which is why we have decided to stick with the $1 \%$ criterion. We would also like to stress that a Bingham fluid is a generalized Newtonian fluid (GNF) meaning that its viscosity is a nonlinear function of the shear-rate. The extra nonlinearity introduced this way may have also affected the quality of our numerical results for this demanding test case.

\section{Figure 7}

To ensure that the results are not affected by the large viscosity in the bi-viscous model, $\mu_{0}$, we have obtained numerical results for a wide range of viscosity ratios. For a given $\mu_{\infty}$, an increase in the viscosity ratio means 
an increase in $\mu_{0}$. As can be seen in Fig. 8 for $B n$ numbers up to 100 , a viscosity ratio larger than $10^{3}$ ensures that the results are independent of $\mu_{0}$. To be on the safe side, in all simulations to be reported next we have relied on a viscosity ratio equal to $10^{6}$.

Figure 8

\section{Results and Discussion}

Having checked different aspects of our numerical scheme, we are now ready to present our new results depicting the effect of a fluid's yield stress on the peristaltic transport of rigid particle(s). As mentioned above, in the results to be presented in the next section we have tacitly assumed that the computational domain comprises just one wavelength. Also, all particles are assumed to be of the same density as that of the fluid. We have been able to obtain converged results over a wide range of the peristaltic wave parameters. In fact, our LBM/SPM has enabled us to easily obtain converged results for the two- and four-particle scenarios, not reported before even for Newtonian fluids [27].

\subsection{One-Particle Case}

Figure 9 shows the effect of the confinement ratio, $\beta$, on the average axial velocity for a single initially centered particle at different $B n$ numbers. For a given $a$, an increase in the confinement ratio means an increase in the particle's diameter. Figure 9 shows that, for Newtonian fluids, the average velocity is increased by an increase in $\beta$. In Ref. 18 it has been shown that, for Newtonian fluids, an interaction between the particle with the wake of its periodic copy can drift the main particle further forward. And, the wake becomes stronger the larger the size of the particle. For viscoplastic fluids, however, the average velocity is predicted to pass through a dip when $\beta$ is increased (see Fig. 9). Figure 10 shows that the Bingham number also affects the instantaneous velocity at a typical $\beta=0.4$. As can be seen in this figure, the particle translates back and forth in the axial direction although the motion is steady in a periodic sense so that there is a net displacement in the direction of the wave's propagation. According to Fig. 10, the backward velocity is significantly increased when the Bingham is increased and this translates itself into a drop in the average velocity as compared with its Newtonian counterpart (i.e., a Newtonian fluid having the same plastic viscosity, $\mu_{p}$ ).

\section{Figure 9}

\section{Figure 10}

Figure 11 shows that the quantitative effect of $B n$ number depends on the Reynolds number although the general trend remains intact. That is, by an increase in the Bingham number, the velocity starts to rise at any given Reynolds number (even at $\mathrm{Re}=1$ ) before passing through a peak and then it approaches an asymptotic value at sufficiently large $B n$ numbers. But, what is more interesting is the finding that while for Newtonian fluids (i.e., for $\mathrm{Bn}=0$ ), an increase in the Reynolds number lowers the velocity of the particle, for sufficiently large $B n$ numbers the largest velocity corresponds to the largest Reynolds number (see Fig. 11). This notion can better be seen in Fig. 12 which shows that, while for Newtonian fluids the particle's velocity is decreased by an increase in $R e$, for viscoplastic fluids it increases by an increase in the Reynolds number. Also, there exists a threshold Reynolds number (roughly 2.5 for this set of parameters) beyond which, yield-stress induce a larger velocity to the particle. This also suggests that, while transporting particles suspended in a viscoplastic fluid, it is better to work at larger Reynolds numbers, if possible.

Figure 11 


\section{Figure 12}

To interpret the above results, we can resort to the morphology of the plugs formed in the domain and how they are affected by the particle. Figure 13 shows typical results obtained for this purpose. The first row in this figure shows results when there is no particle present in the channel, and the second row shows the effect of the particle on the plugs. As can be seen in this figure, the size and shape of the plug is affected by the presence of the particle. Interestingly, the particle is seen to enter the plug at certain times and to exit at certain other times inferring that there is a difference between the velocity of the plug and the velocity of the particle. As mentioned above, based on current knowledge obtained for Newtonian fluids [18], the particle interacts with the wake of its periodic copy to such an extent that the main particle drifts itself further forward. This mechanism is also operational for the viscoplastic fluids although the plugs formed along the centerline for such fluids (see Fig. 13) interfere with the wake in a non-trivial fashion. And, this might be partially responsible for the dip in the particle's velocity when $\beta$ is increased (see Fig. 9). The Reynolds number is known to affect the plug's morphology [22], and so it can affect the particle's transport quantitatively although the general picture remains virtually intact [see Fig. 11].

\section{Figure 13}

Figure 14 shows the effect of the wave number, $\alpha$, on the average axial velocity of the particle. This figure clearly shows that for any given $B n$, the axial velocity of the particle is increased by an increase in the wave number (or, equivalently, by a decrease in the wavelength). The strong effect of the wave number for viscoplastic fluids has been reported in our recent work [22]. But, what is more striking is the notion that there appears to exist a critical wave number (roughly 0.35 for this set of parameters) below which fluid's yield stress has a retarding effect on the particle's transport but above which it has an accelerating effect. (This is a significant finding which has technological implications in the pump industry.) The nontrivial effect of the wave number on the flow characteristics can again be attributed to its effect on the shape, size, and location of the plugs formed anywhere in the domain.

\section{Figure 14}

Figure 15 shows how the amplitude ratio, $\phi$, affects the particle's transport in a viscoplastic fluid. From this figure it can be seen that for Newtonian fluids the time-averaged axial velocity of the particle is monotonically increased when the amplitude ratio is increased until a plateau is reached (say, at roughly $\phi=0.65$ ). For nonzero Bingham numbers, however, the velocity of the particle is decreased if the amplitude ratio exceeds a critical value (roughly $\phi \approx 0.45$ for $\mathrm{Bn}=5$ ). To explain this rather unexpected behavior we can resort to the instantaneous axial velocity and lateral position of the particle as shown in Fig. 16. This figure shows that whereas at $B n=0$ and $B n=1$ the particle remains aligned with the centerline of the channel, at $B n=5$ it is displaced far from the centerline. This peculiar behavior can also be seen in the instantaneous axial velocity of the particle as compared with the case of $B n=0$ and $B n=1$. This can better be seen in Fig. 17 which shows the streamline pattern for the case of $B n=5$. From this figure it can be concluded that, at this particular Bingham number (i.e., at $\mathrm{Bn}=5$ ) there are large vortices formed at the largest area of the channel. The particle is obviously affected by these vortices in such a way that it is dislocated from the centerline. This gives rise to an asymmetry in the morphology of the plug further complicating the situation (see Fig. 17). The particle is also seen to start rotating around its center-of-mass for this particular set of parameters. In Fig. 17, a triangular marker on the periphery of the particle shows how the particle is rotating while being simultaneously transported along the channel.

\section{Figure 15}

\section{Figure 16}




\section{Figure 17}

It is worth mentioning that in all of the above simulations the particle was initially located at the centerline of the channel $(\eta=0)$. Figure 18 shows the case in which the particle is initially located off-center at either $\eta=0.2$ or $\eta=0.7$. As can be seen in this figure, off-center particles oscillate laterally while being shifted towards the channel's centerline with an amplitude which is decaying with time. That is, regardless of its initial positioning, the particle eventually lodges itself at the channel's centerline. Interestingly, the particle needs lesser time to reach the centerline of the channel if the suspending fluid is viscoplastic (see Fig. 18). The large oscillations of the particle in the course of its transport is probably due to a fluctuation in the pressure field which exerts a side force of the same nature on the particle thereby causing it to oscillate-a phenomenon which has also been reported for Newtonian fluids in Ref. 18. Figure 19 shows the effect of the $B n$ number on the instantaneous axial velocity of the particle. A fluctuation in the velocity is evident in this figure. The amplitude of the fluctuations is seen to be larger for the particle which is farther away from the centerline, but it approaches an asymptotic value as time progresses-with an average which depends on Bn (see Fig. 19). The Bingham number also affects the particle's angular position, as can be seen in Fig. 20.

\section{Figure 18}

\section{Figure 19}

Figure 20

\subsection{Multi-Particle Case}

For the two-particle case, three different initial arrangement could be envisaged, as depicted in Fig. 21. Table 1 shows the coordinates of the center-of-mass for each particle in each initial configuration.

\section{Figure 21}

\section{Table 1}

Figure 22 shows the numerical results obtained for the lateral position of the two particles for Case A. This figure shows that in a Newtonian fluid the two particles remain along the centerline for quite some time. For some reasons, however, they suddenly leave the centerline and stay at a fixed distance far from it while oscillating laterally and rotating in the opposite directions (see Fig. 22a). This notion can better be seen in Fig. 23 which also shows the streamline pattern. As far as we are aware, there is no such results published in the open literature even for Newtonian fluids, although it should be conceded that in a recent work dealing with Newtonian fluids it has been speculated that the center state might be unstable [29]. In fact, the present work appears to be the first numerical proof of this amazing speculation. (A linear stability analysis can reveal the reason behind this unexpected finding, which can be the subject of an ensuing work.) The yield-stress can suppress this phenomenon as can be seen at $B n=5$ where the particles remain close to the centerline at all times (see Fig. 24a). They also stop rotating at $B n=5$ while being transported further downstream, as can be seen in Fig. 24b.

\section{Figure 22}

Figure 23

Figure 24 
Figure 25 shows the numerical results obtained for the Case B. These figures show that in a Newtonian fluid initially off-centered particles get closer to each other but they never reach the centerline with the final configuration being symmetric. Interestingly, the situation remains virtually the same even in the Bingham fluid with the only difference being that the amplitude of the fluctuations is slightly smaller as compared with the Newtonian case.

\section{Figure 25}

Figure 26 show the numerical results obtained for the Case C. In a Newtonian fluid, the two particles again approach each other in a rather asymmetric fashion but settle at an equal distance above and below the centerline. In yield-stress fluids, however-although both particles eventually settle at the centerline-at the early stages of the peristaltic motion, particle 1 jumps from the upper half of the channel to the lower half of the channel in a rather erratic fashion (see Fig. 26b).

\section{Figure 26}

All in all, these figures show that the off-center positioning of the two-particles play a key role in their eventual fate while suspended in a viscoplastic fluids. In fact, depending on the Bingham number, only for certain set of off-center positioning, the particles may eventually align themselves with the centerline of the channel. The strong influence of the yield stress on the particle transport can be attributed to the formation of plugs along the centerline. Formation of such plugs can best be seen in Fig. 27 which shows the situation at dimensionless time $\Lambda=40$.

\section{Figure 27}

The flow characteristics are expected to become much more complicated when more than two particles are involved. For the four-particle scenario, we have tried two different initial configurations for our simulations. They are shown graphically in Fig. 28 and are denoted by Case A and Case B. In Table 2 the coordinates of these particles have been given for each initial setting.

\section{Figure 28}

\section{Table 2}

The simulation are done for a typical $\operatorname{Re}=5, \alpha=0.5, \beta=0.2$, and $\phi=0.25$. Figure 29 shows the numerical results obtained for Case $A$ in a Newtonian fluid and also in a Bingham fluid. As can be seen in this figure, in the Newtonian fluid, the upper and lower particles approach each other as soon as the flow starts, but eventually settle at a fixed distance away from the centerline. This is tantamount to saying that the two particles in each side of the centerline always remain at the same side no matter how long the simulation is run. For the Bingham fluids, however, we are witnessing a rather erratic type of particle movement so that certain particles (e.g., particle 2) move to the other side of the centerline (see Fig. 29). Still, the overall picture is that the fluid's yield stress causes the particles to get closer to each other. The particles stick to each other and act like a larger solid body which continuously changes its shape and configuration as it moves further downstream (see Fig. 30).

\section{Figure 29}

\section{Figure 30}

Figure 31 shows similar results obtained for the Case B in a Newtonian fluid and also in a Bingham fluid. As can be seen in Fig. 31, as soon as the motion starts, in a Newtonian liquid the four particles start aligning themselves with the centerline of the channel and stay there for prolonged times. But, at a dimensionless time of roughly 400 the particle's configuration become unstable and suddenly start to oscillate and drift off center. For 
the Bingham fluid we are witnessing a scenario quite similar to the Case A. That is, the four particles approach each other and move like a larger solid body with significant inter-particle movement, as can be seen in Fig. 32.

\section{Figure 31}

\section{Figure 32}

\section{Concluding Remarks}

In this work, we have investigated the role played by a fluid yield stress on the peristaltic transport of rigid circular particles in a planar channel for the case of "free-pumping". For ease of analysis, the fluid and the particle have been assumed to be of the same density. We have relied on the LBM method combined with the SPM method for the simulations. We have been able to obtain converged results at non-zero Reynolds numbers for sufficiently large Bingham numbers over a wide range of wave parameters. Based on the numerical results obtained in the present work, we have reached to the following conclusions:

- While for Newtonian fluids the peristaltic transport of a single rigid particle is facilitated by an increase in the confinement ratio, for viscoplastic fluids the confinement ratio should be larger than a critical value for this to happen.

- For any given confinement ratio, a fluid's yield stress lowers the particle's axial velocity, as compared with its Newtonian counterpart.

- An increase in the Reynolds number can increase or decrease particle's velocity depending on the Bingham number.

- There exists a critical wave number above which a fluid's yield stress can boost particle's transport.

- For viscoplastic fluids there exists an amplitude ratio (which depends on the Bingham number) at which the time-averaged velocity of the particle is maximized.

- Off-center particles always tend to align themselves with the channel's centerline, and this is facilitated by an increase in the fluid's yield stress.

- Depending on the Bingham number, an object initially located at the centerline might drift off-axis and start rotating.

To interpret such intriguing results, we have resorted to effect of fluid's yield stress on the shape, size, and location of the plug(s) formed in the channel. Formation of such plugs affects particle's motion which in return affects the shape and size of the plugs at the first place. So, in a sense, we are dealing with a complicated fluidsolid interaction (FSI) problem. In practice, such effects manifest themselves through the rise of a threshold which is found to exist for virtually all parameters involved in the problem (e.g., the wave number). This also suggests that through a judicial selection of the wave parameters it is possible to enhance particle's axial velocity (in a time-averaged sense) when the fluid surrounding the particle is viscoplastic. In addition, based on our numerical results, the yield stress in a fluid can be useful for peristaltic transport of solid particles provided that the Reynolds number is sufficiently large. For the case of two or more particles, the interaction between the particles with each other and also with the fluid and the wall complicates the picture enormously. Fortunately, our code could easily handle such cases. Such two- and four-particle scenarios are of particular importance in physiological systems where a kidney stone might be broken down (non-intrusively) to smaller particles. One would then be interested in knowing if the emergence of such smaller particles boosts or hinders the overall transport. Limited data obtained using our code for the two-particle case shows that depending on the initial positioning of the particles with respect to the channel's centerline, different scenarios might emerge as to the eventual fate of the particles. Obviously, this is an interesting subject which warrants further study on its own right. Work is currently going on in our research group to further elaborate on this intriguing issue.

A major finding of the present work is that the configuration corresponding to initially-centered particles may become unstable at prolonged times. The assumption pervading the literature is that particles initially located at 
the centerline of the channel always remains there no matter how long the simulation is run. That is to say that, the centerline is normally deemed to be a stable position in peristaltic flows of particulate systems. Our numerical results support this idea as long as a single particle is involved. This is not necessarily true when more than one particle is involved. That is, when two or more particles are initially located along the centerline and are subjected to peristaltic motion, the center position may easily become unstable. Our preliminary results suggest that the yield stress can have a strong effect on this phenomenon. An independent work is obviously needed to delineate the mechanism(s) behind this kind of instability. A linear stability analysis would be a good next step to extend the current work.

\section{Acknowledgement:}

The authors wish to express their sincerest thanks to Iran National Science Foundation (INSF) for supporting this work under contract number 95815139.

\section{Nomenclature:}

Half-height of the unperturbed channel

Amplitude of the travelling wave

Wavelength

Wave speed

Longitudinal coordinate in the laboratory frame

Longitudinal coordinate in the laboratory frame

Height of a point on the wall in the laboratory frame

Time

Lateral coordinate of the upper wall

Lateral coordinate of the lower wall

Half of the maximum height of the channel

Velocity vector of the fluid

Fluid's density

Solid's density

Isotropic pressure

Extra stress tensor

Rate-of-deformation tensor

Fluid's yield stress

Viscosity of the un-yielded region in bi-viscous Bingham model

Viscosity of the yielded region

Second invariant of rate-of-deformation tensor

Critical shear rate of the bi-viscous Bingham model

Wave number

Fraction ratio

Amplitude ratio

Bingham number

Reynolds number

Period of the travelling wave

Velocity vector of each point of the particles

Axial velocity of the particles

Instantaneous axial dimensionless velocity of the particles

Time-averaged axial dimensionless velocity of the particles

Period of oscillations for a particle

Particle's radius

Position of particle 
$\delta \quad$ Interfacial thickness of the solid particles

$\varphi \quad$ Density profile

$\xi \quad$ Dimensionless longitudinal coordinate in the laboratory frame

$\eta \quad$ Dimensionless lateral coordinate in the laboratory frame

$\Lambda \quad$ Dimensionless time

$\beta \quad$ Volume fraction

$\sigma \quad$ Density ratio

\section{References:}

[1] T.W. Latham, Fluid motions in a peristaltic ump, M.Sc Thesis, M.I.T., Cambridge, Mass., 1966.

[2] J.C. Burns, and T. Parkes, Peristaltic motion, J. Fluid Mech., 29 (1967) 731-748.

[3] Y.C. Fung, and C.S. Yih, Peristaltic transport, J. Appl. Mech., 35 (1968) 669-675.

[4] A.H. Shapiro, M.Y. Jaffrin, and S.L. Weinberg, Peristaltic pumping with long wavelengths at low Reynolds number, J. Fluid Mech., 37 (1969) 799-825.

[5] T.F. Zien, and S. Ostrach, A long wave approximation to peristaltic motion, J. Biomech., 3 (1970) 6375 .

[6] S.L. Weinberg, E.C. Eckstein, and A.H. Shapiro, An experimental study of peristaltic pumping, J. Fluid Mech., 49 (1971) 461-479.

[7] Y.C. Fung, and C.S. Yih, Comparison of theory and experiment in peristaltic transport, J. Fluid Mech., 47 (1971) 93-112.

[8] M.Y. Jaffrin, A.H. Shapiro, Peristaltic pumping., Ann. Rev Fluid Mech., 3 (1971) 13-36.

[9] S. Takabatake, and K. Ayukawa, Numerical study of two dimensional peristaltic flows, J. Fluid Mech., 122 (1982) 439-465.

[10] C. Pozridikis, A study of peristaltic flows, J. Fluid Mech.,180 (1987) 515-527.

[11] T.K. Hung, and T.D. Brown, Solid-particle motion in two-dimensional peristaltic flows, J. Fluid Mech., 73(1) (1976) 77-96.

[12] M.R. Kaimal, Peristaltic pumping of a Newtonian fluid with particles suspended in it at low Reynolds number under long wavelength approximations, Journal of Applied Mechanics, 45(1) (1978) 32-36.

[13] L.M. Srivastava, V.P. Srivastava, Peristaltic transport of a particle-fluid suspension, J. Biomech. Eng., 111(2) (1989) 157-165.

[14] C.J. Misra, K.S. Pandey, Peristaltic transport of a particle-fluid suspension in a cylindrical tube, Comput. Math Appl., 28 (1994) 131.

[15] K.S. Mekheimer, E.F. El-Shehawey, A.M. Elaw, Peristaltic motion of a particle-fluid suspension in a planar channel, Int. J. Theor. Phys., 37 (1998) 2895.

[16] J. Jiménez-Lozano, M. Sen, and P.F. Dunn, Particle motion in unsteady two-dimensional peristaltic flow with application to the ureter, Phys. Rev.E,79(4) (2009) 041901.

[17] L. Fauci, Peristaltic pumping of solid particles, Comp. \& Fluids, 21(4) (1992) 583-598.

[18] K. Connington, Q. Kang, H. Viswanathan, A. Abdel-Fattah, and S. Chen, Peristaltic particle transport using the lattice Boltzmann method, Phys. of Fluids, 21(5) (2009), 053301-16. 
[19] J. Chrispell, L. Fauci, Peristaltic Pumping of Solid Particles Immersed in a Viscoelastic Fluid, Math. Model. Nat. Phenom., 6(11) (2011) 67-83.

[20] Y. Nakayama, and R. Yamamoto, Simulation method to resolve hydrodynamic interactions in colloidal dispersions. Physical Review E, 71(3) (2005) 036707-7.

[21] S.R. Jafari, Y. Yamamoto, and M. Rahnama, Lattice-Boltzmann method combined with smoothedprofile method for particulate suspensions. Physical Review E, 83(2) (2011) 026702-7.

[22] N.P. Khabazi, S.M. Taghavi, K. Sadeghy, Peristaltic flow of Bingham fluids at large Reynolds numbers: A numerical study, J. Non-Newtonian Fluid Mech., 227 (2016) 30-44.

[23] C.W. Macosko, Rheology: Principles, Measurements and Applications, first ed., Wiley VCH, 1994.

[24] C.R. Beverly, R.I. Tanner, Numerical analysis of three-dimensional Bingham plastic flow, J. NonNewt. Fluid Mech., 42 (1992) 85-115.

[25] R. Glowinski, T.-W. Pan, T.I. Hesla, D.D. Joseph, A distributed Lagrange multiplier/fictitious domain method for particulate flows. International Journal of Multiphase Flow, 25(5) (1999) 755-794.

[26] Z. Feng, and E. E. Michaelides, The immersed boundary-lattice Boltzmann method for solving fluidparticles interaction problems, J. of Computational Physics, 195(2) (2004) 602-628.

[27] N.P. Khabazi, Peristaltic flow of Bingham fluids: a numerical study, PhD thesis, University of Tehran, in progress.

[28] V. Stobiac, P.A. Tanguy, and F. Bertrand, Investigation of the accuracy of the extrapolation method for the lattice Boltzmann simulation of viscous fluid flow in a Maxblend impeller system. Computers \& Chemical Engineering, 60 (2014) 112-123.

[29] D. Takagi, N. J. Balmforth, Peristaltic pumping of rigid objects in an elastic tube, J. Fluid Mech., 672 (2011) 219-244.

\section{List of Figures:}

Figure 1: Schematic showing the flow domain and its geometrical parameters.

Figure 2: A schematic showing smooth variation of a particle's density profile.

Figure 3: A comparison between our numerical results with the results obtained by Facuci [17] and Connington et al [18] for peristaltic transport of a rigid cylindrical particle suspended in a Newtonian fluid: (a) dimensional axial location, (b) and dimensional axial velocity $(\operatorname{Re}=1, \alpha=0.25, \varphi=0.16, \beta=0.4)$.

Figure 4: Effect of the domain size on the average axial velocity of a single particle suspended in a Newtonian fluid at different confinement ratios $(\operatorname{Re}=1, \alpha=0.25, \varphi=0.16)$.

Figure 5: The effect of domain size, shown by the darker color, on the distance between the main particle and its periodic copy when just one particle is involved.

Figure 6: Effect of the mesh size on the time-averaged axial velocity at different confinement ratios (left plot) and different Bingham numbers (right plot) obtained at: (a) $B n=5, \operatorname{Re}=1, \alpha=0.25, \varphi=0.16$, (b) $\operatorname{Re}=10, \alpha=0.2, \beta=0.4, \varphi=0.25$.

Figure 7: Spatial convergence study. (a) Schematic of the problem (b) Effect of the lattice spacing on the spatial error.

Figure 8: Effect of the viscosity ratio, $\mu_{0} / \mu_{\mathrm{p}}$, in the bi-viscous Bingham model on the average axial velocity of the particle $(\operatorname{Re}=3.125, \alpha=0.25, \varphi=0.16, \beta=0.4)$.

Figure 9: Effect of the confinement ratio on the axial velocity obtained for different Bingham numbers for a single particle suspended in a bi-viscous Bingham fluid $(\operatorname{Re}=1, \alpha=0.25, \varphi=0.16)$. 
Figure 10: Effect of the Bingham number on the instantaneous axial velocity at different dimensionless times obtained for a single particle suspended in a bi-viscous Bingham fluid $(\operatorname{Re}=1, \alpha=0.25, \beta=0.4, \varphi=0.16)$.

Figure 11: Effect of the Bingham number on the average axial velocity of a single particle obtained at three different Reynolds numbers $(\alpha=0.2, \varphi=0.25, \beta=0.4)$.

Figure 12: Effect of the Reynolds number on the time-averaged axial velocity of the particle obtained at $\alpha=0.2, \beta=0.4, \varphi=0.2$ for different $B n$ numbers.

Figure 13: The effect of a single solid particle on the morphology of plug(s) formed at $\operatorname{Re}=5, \mathrm{Bn}=20, \alpha=$ $0.5, \beta=0.2, \phi=0.3$ at different dimensionless times.

Figure 14: Effect of the wave number on the average axial velocity of the suspended particle obtained at three different Bingham numbers $(\operatorname{Re}=0.1, \varphi=0.2, \beta=0.4)$.

Figure 15: Effect of the amplitude ratio on the average axial velocity of the particle obtained at three different Bingham numbers $(\operatorname{Re}=5, \alpha=0.5, \beta=0.2)$.

Figure 16: Effect of the Bingham number on the instantaneous particle's velocity (left plot) and its lateral position (right plot) obtained for: $\operatorname{Re}=5, \alpha=0.5, \beta=0.2, \varphi=0.5$, ).

Figure 17: Streamline pattern and particle's rotation obtained at $\operatorname{Re}=5, \alpha=0.5, \beta=0.2, \varphi=0.5, B n=5$ for different dimensionless times: $\Lambda=0.5,1.5,3.5,7.5,23,24,25,26,27,28,29,30,31,32,33,34$ (from left to right).

Figure 18: Effect of off-center positioning on the particle's lateral position obtained at $\operatorname{Re}=5, \alpha=0.5, \beta=0.2, \varphi=0.25:$ (a) $\eta_{0}=0.2$, (b) $\eta_{0}=0.7$.

Figure 19: Effect of off-center positioning on the particle's instantaneous velocity at $\operatorname{Re}=5, \alpha=0.5, \beta=0.2, \varphi=0.25:$ (a) $\eta_{0}=0.2$, (b) $\eta_{0}=0.7$.

Figure 20: Effect of off-center initial positioning on angular rotation of particle obtained at $\operatorname{Re}=5, \alpha=0.5, \beta=0.2, \varphi=0.25:$ (a) $\eta_{0}=0.2$, (b) $\eta_{0}=0.7$.

Figure 21: Schematic showing the initial location of the two particles in the channel based on Table $1(\operatorname{Re}=$ $5, \alpha=0.5, \beta=0.2, \phi=0.25)$.

Figure 22: (a) lateral position, and (b) angular position of the particles in a Newtonian fluid as a function of time for Case A obtained at $\operatorname{Re}=5, \alpha=0.5, \beta=0.2, \phi=0.25$.

Figure 23: Particle's location and rotation for $\operatorname{Re}=5, \alpha=0.5, \beta=0.2, \varphi=0.25, B n=0$ at different dimensionless times.

Figure 24: Effect of the Bingham number on (a) lateral position, and (b) angular position of particle 1 for Case A obtained at $\operatorname{Re}=5, \alpha=0.5, \beta=0.2, \phi=0.25$.

Figure 25: The lateral position of the particles as a function of time for Case $\mathrm{B}$ obtained at $\operatorname{Re}=5, \alpha=0.5, \beta=$ $0.2, \phi=0.25$ : (a) $B n=0$, and (b) $B n=5$.

Figure 26: The lateral position of the particles as a function of time for $\operatorname{Case} \mathrm{C}$ obtained at $(\operatorname{Re}=5, \alpha=0.5, \beta=$ $0.2, \phi=0.25$ ): (a) $B n=0$, and (b) $B n=5$.

Figure 27: The lateral position of the two particles as a function of time obtained at $\mathrm{Bn}=5$ at dimensionless time $\Lambda=40(\operatorname{Re}=5, \alpha=0.5, \beta=0.2, \phi=0.25)$.

Figure 28: Initial positioning of the particles in the channel.

Figure 29: Effect of the Bingham number on the lateral position of the four particles in Case A configuration.

Figure 30: The position of the particles at different times for Case $\mathrm{A}$ at $B n=5$. 
Figure 31: Effect of the Bingham number on the lateral position of the four particles in Case B configuration.

Figure 32: The position of the particles at different times for Case B at $B n=5$.

\section{List of Tables:}

Table 1: Coordinates of the two particles for three different initial arrangements.

Table 2: Coordinates of the four particles for three different initial arrangements. 


\section{Tables}

Table 1: Coordinates of the two particles for three different initial positioning.

\begin{tabular}{|c|c|c|}
\hline Case & Particle 1 & Particle 2 \\
\hline A & $(+0.25,0.0)$ & $(+0.75,0.0)$ \\
\hline B & $(+0.5,+0.5)$ & $(+0.5,-0.5)$ \\
\hline C & $(+0.75,+0.5)$ & $(+0.25,-0.5)$ \\
\hline
\end{tabular}

Table 2: Coordinates of the four particles for two different initial positioning.

\begin{tabular}{|c|c|c|c|c|}
\hline Case & Particle 1 & Particle 2 & Particle 3 & Particle 4 \\
\hline A & $(+0.75,+0.5)$ & $(+0.75,-0.5)$ & $(+0.25,+0.5)$ & $(+0.25,-0.5)$ \\
\hline B & $(+0.87,+0.5)$ & $(+0.63,-0.5)$ & $(+0.37,+0.5)$ & $(+0.13,-0.5)$ \\
\hline
\end{tabular}




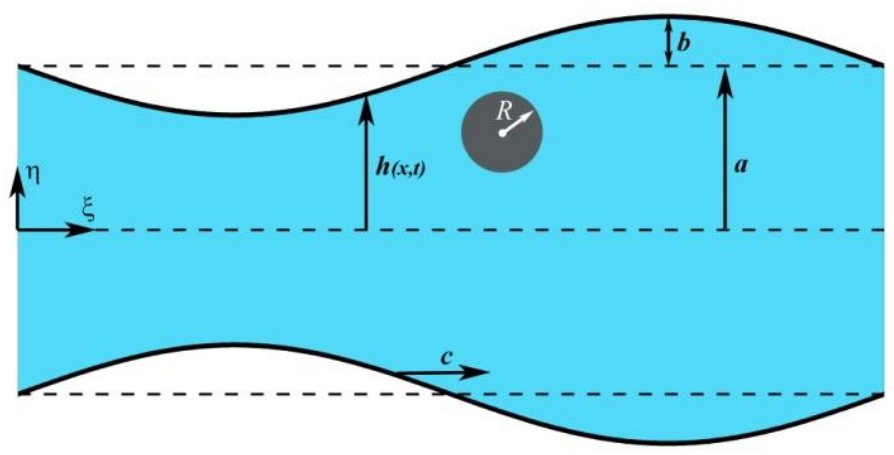

Figure 1

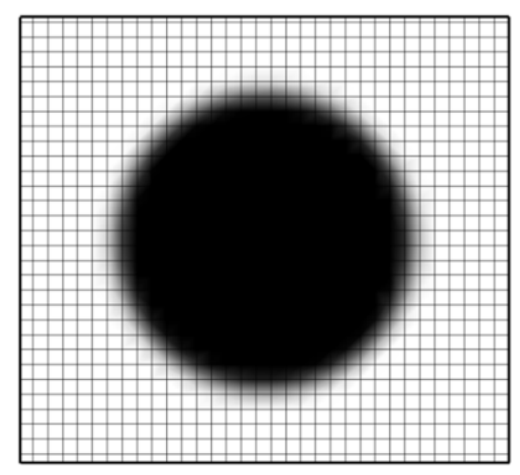

Figure 2

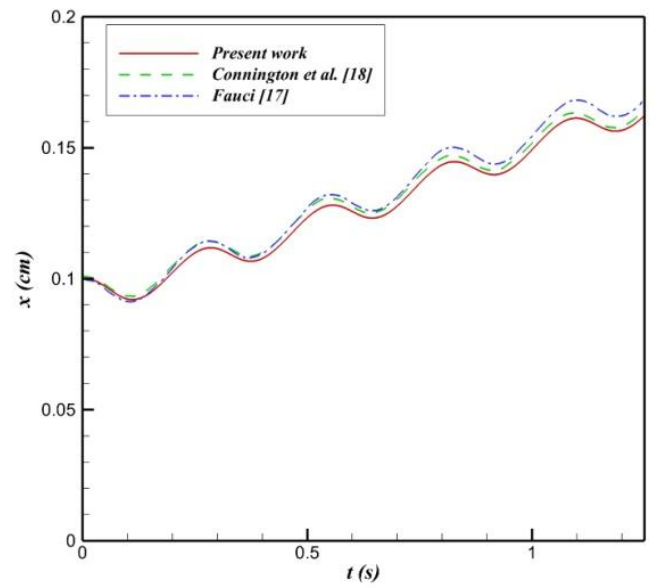

(a)

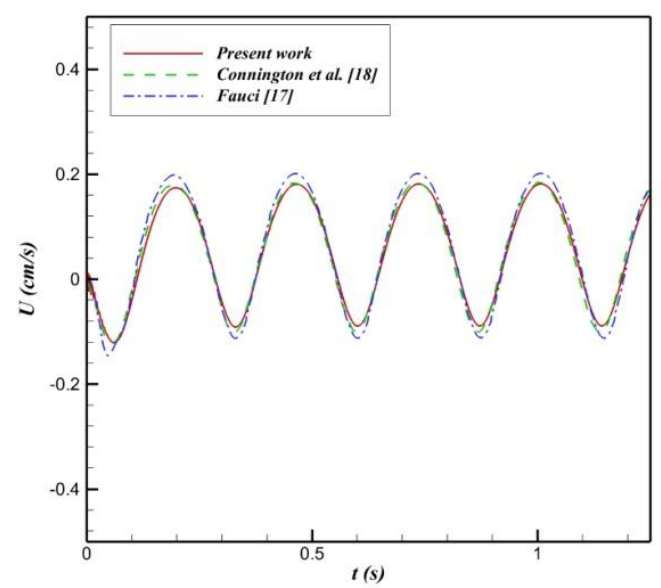

(b)

Figure 3 


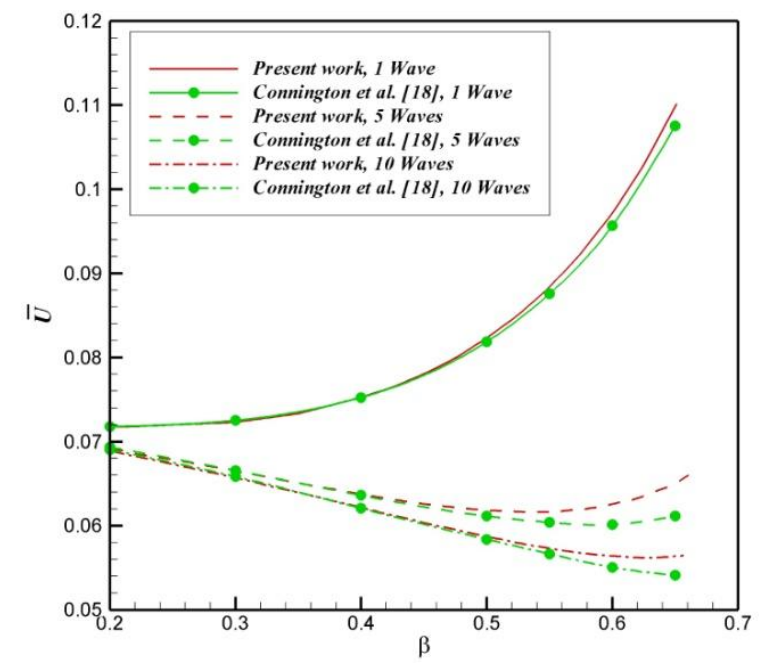

Figure 4
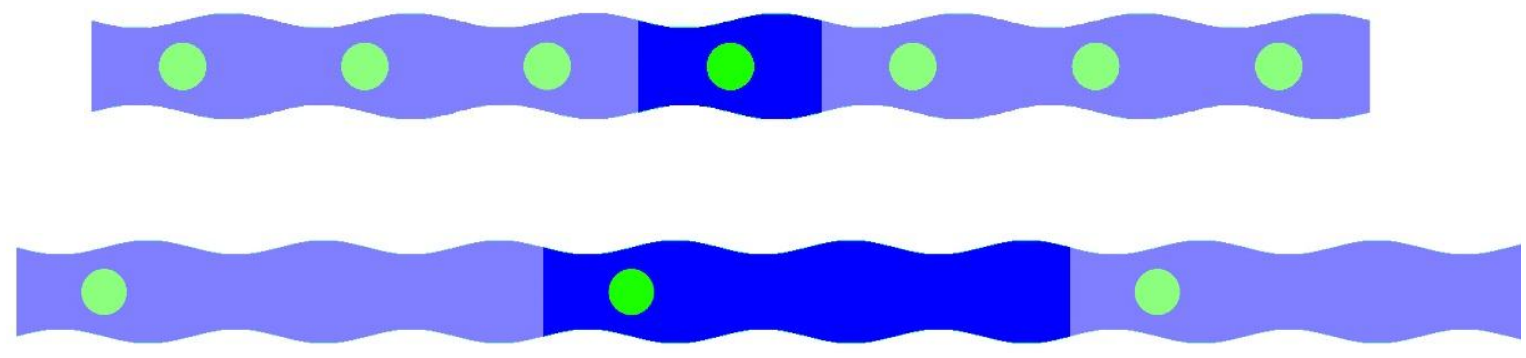

Figure 5

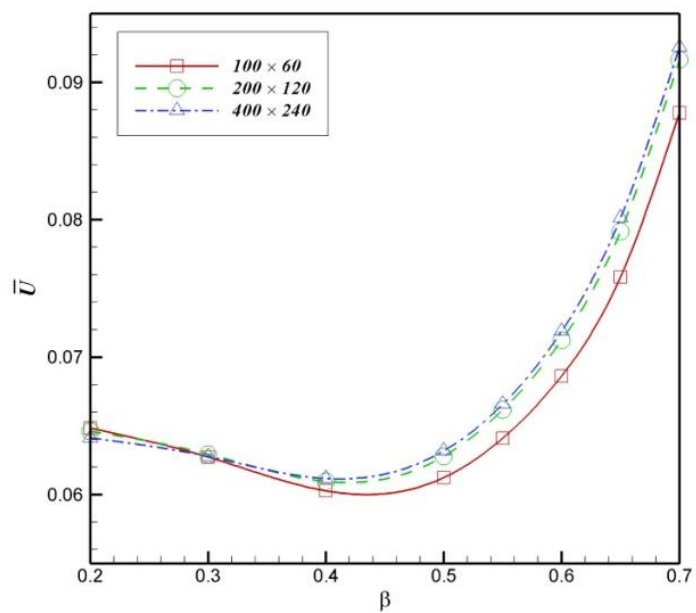

(a)

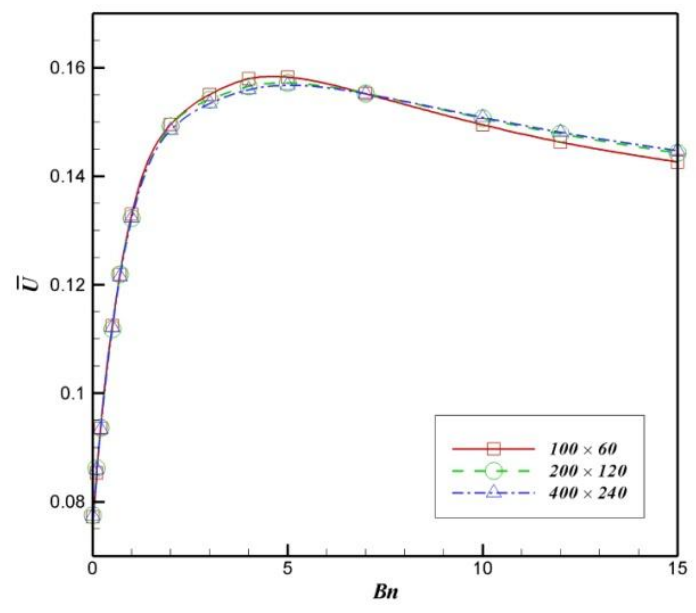

(b)

Figure 6 


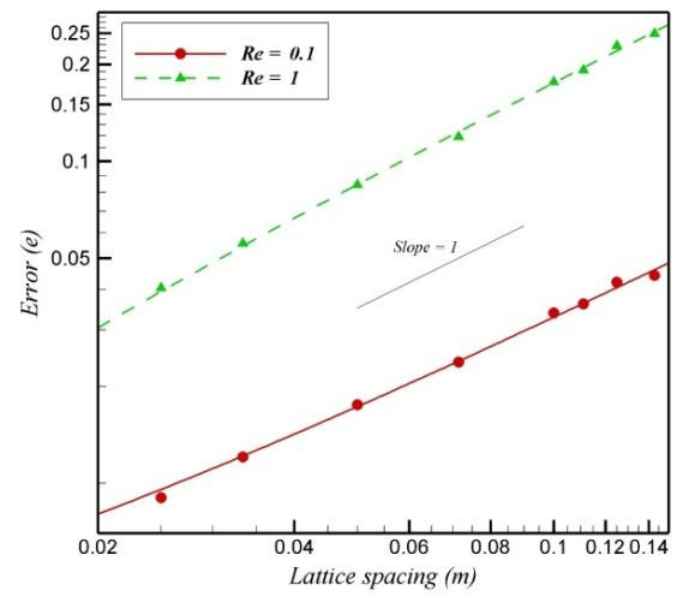

(a)

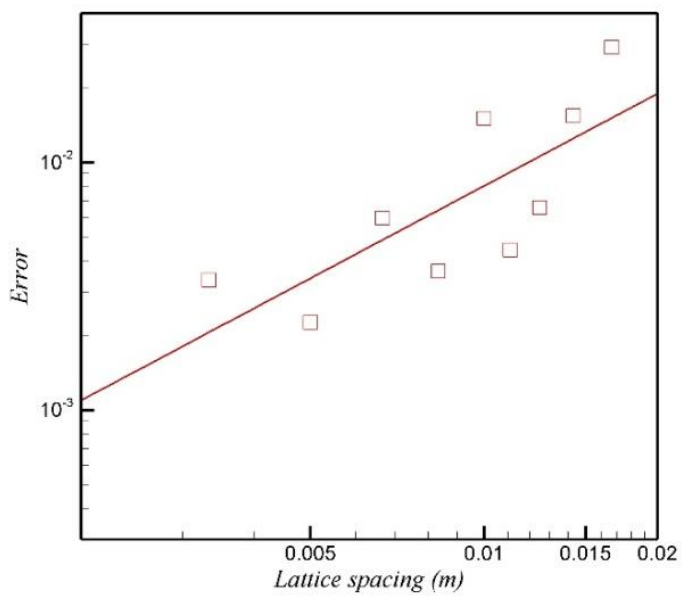

(b)

Figure 7

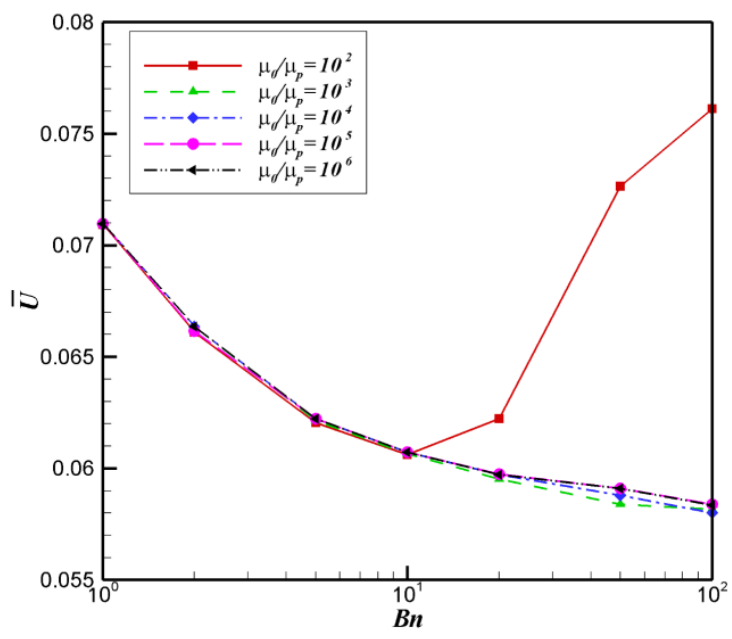

Figure 8 


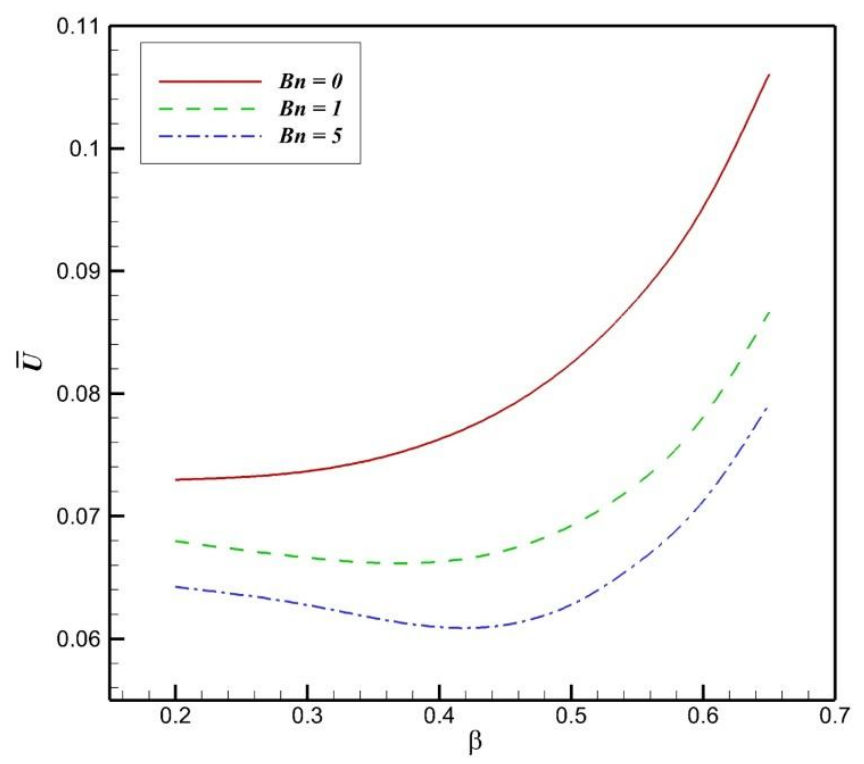

Figure 9

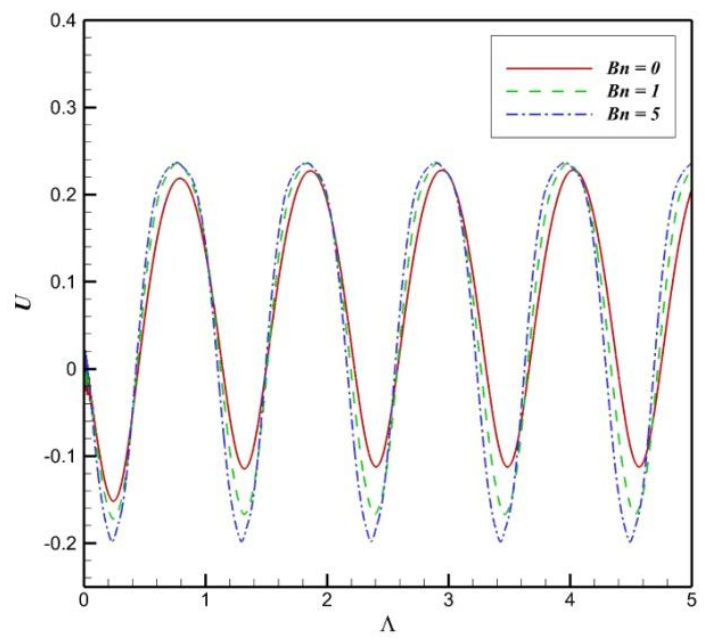

Figure 10 


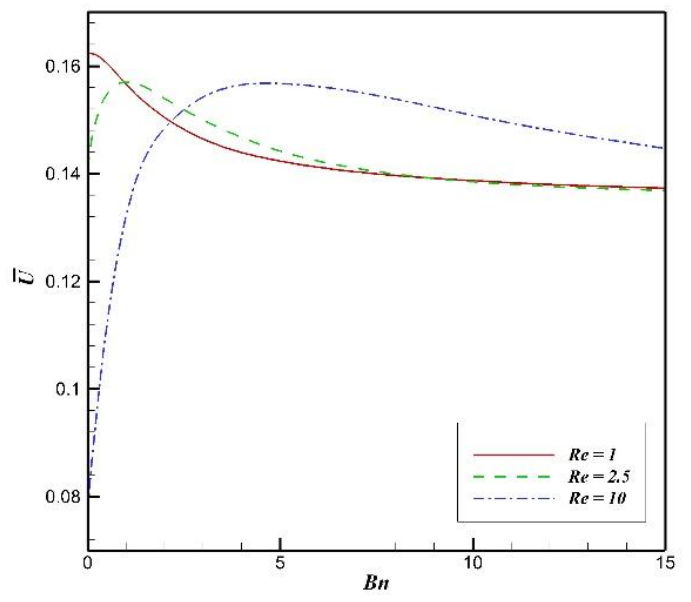

Figure 11

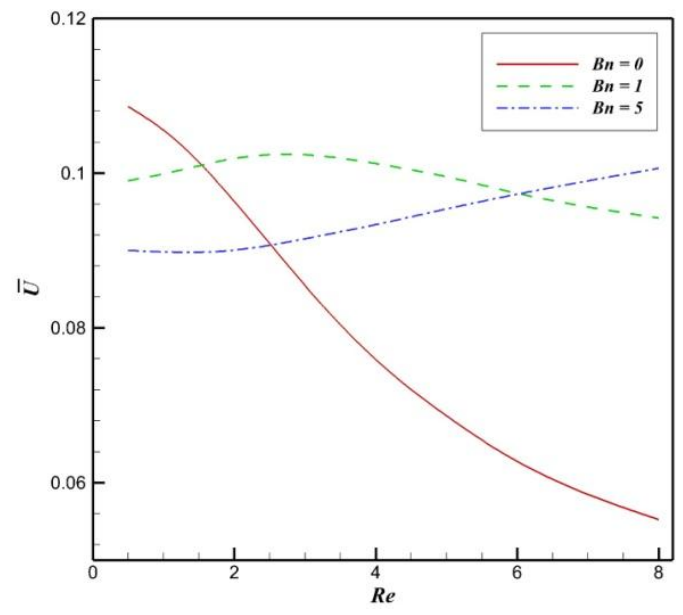

Figure 12 

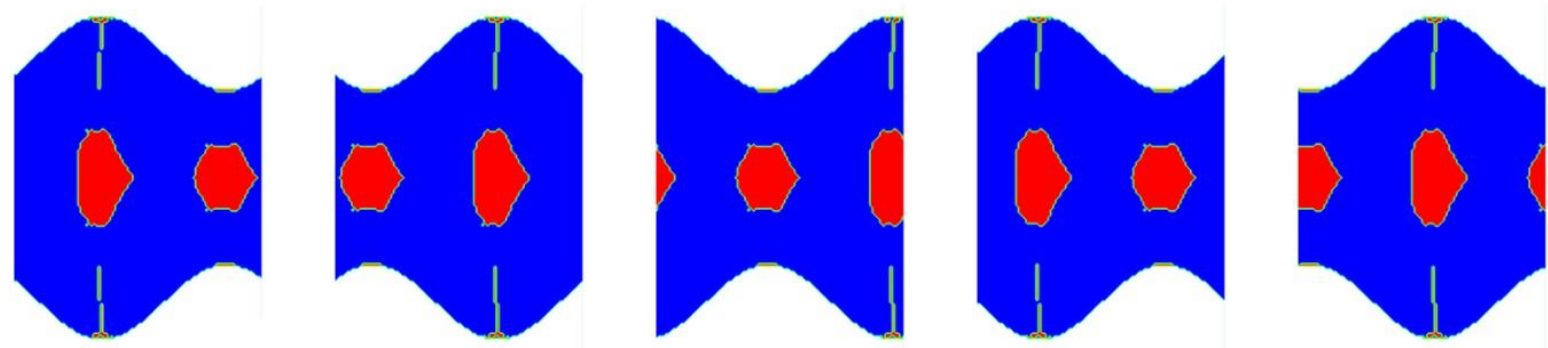

$\Lambda=5.6$

$\Lambda=5.9$

$\Lambda=6.2$

$\Lambda=6.5$

$\Lambda=6.8$
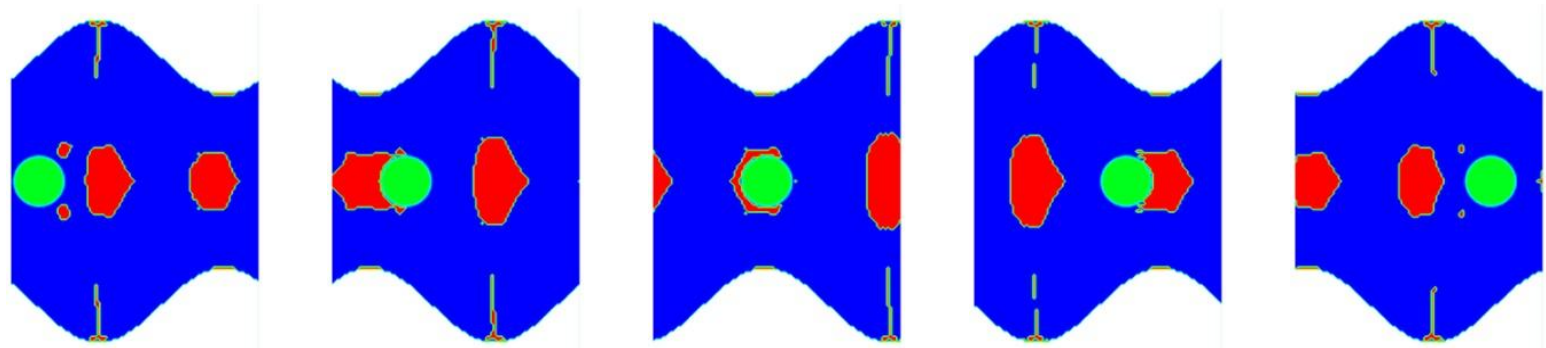

$\Lambda=5.6$

$\Lambda=5.9$

$\Lambda=6.2$

$\Lambda=6.5$

$\Lambda=6.8$

Figure 13

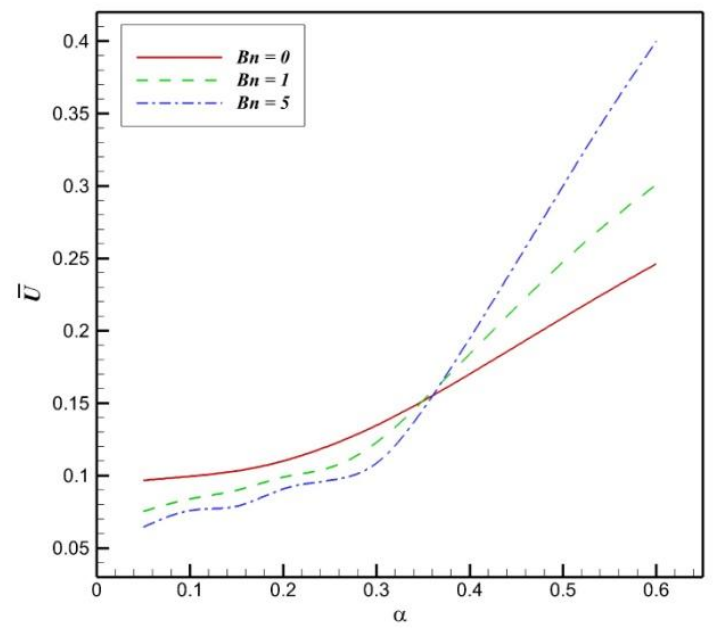

Figure 14 


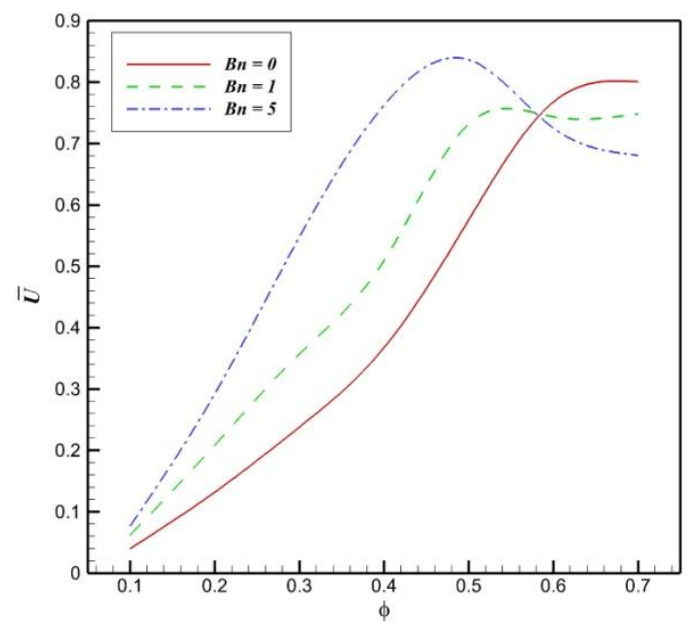

Figure 15

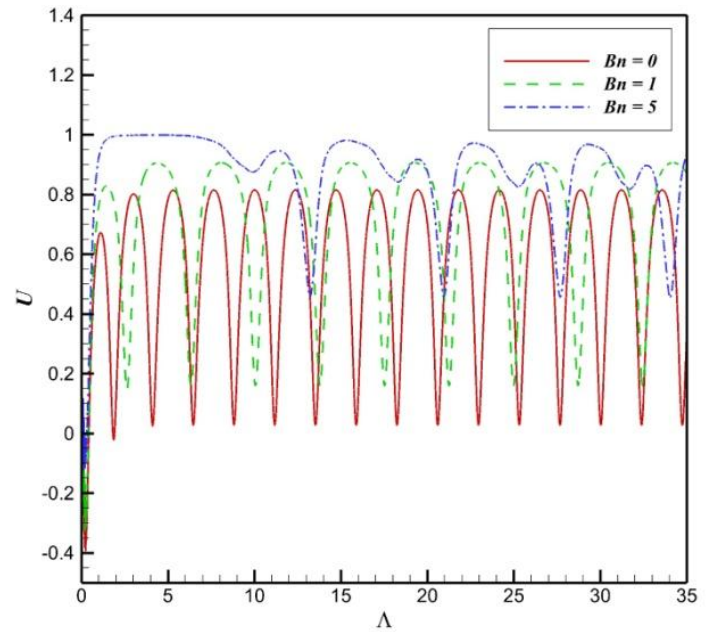

(a)

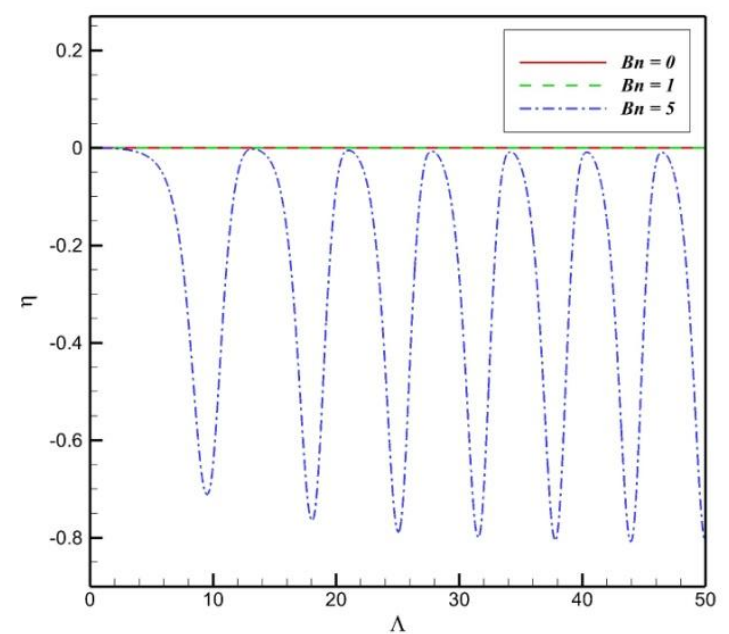

(b)

Figure 16 

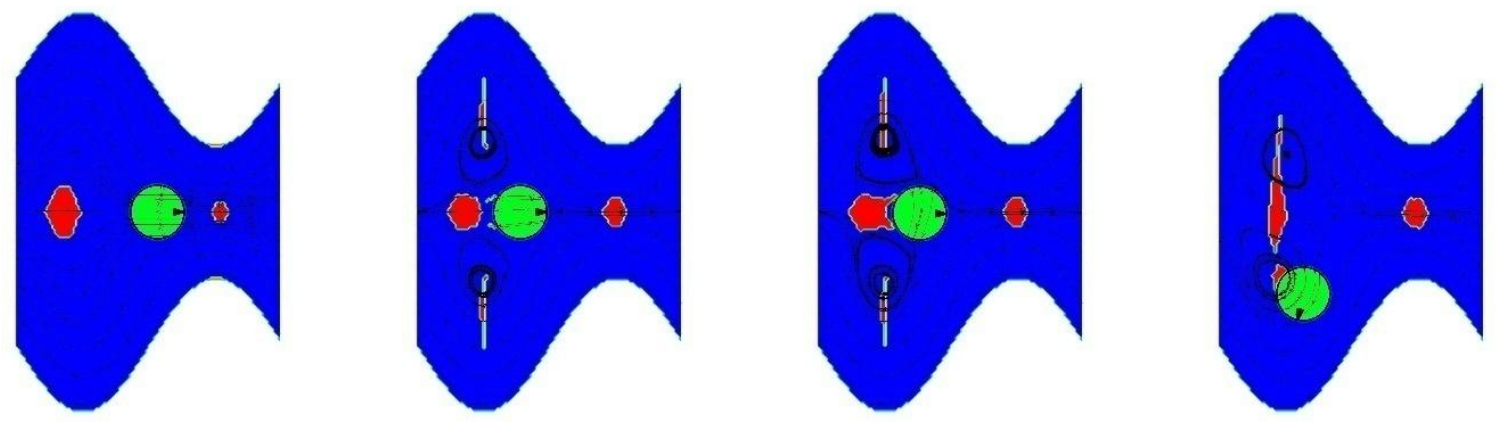

$$
\Lambda=0.5
$$

$$
\Lambda=1.5
$$

$$
\Lambda=3.5
$$

$$
\Lambda=7.5
$$
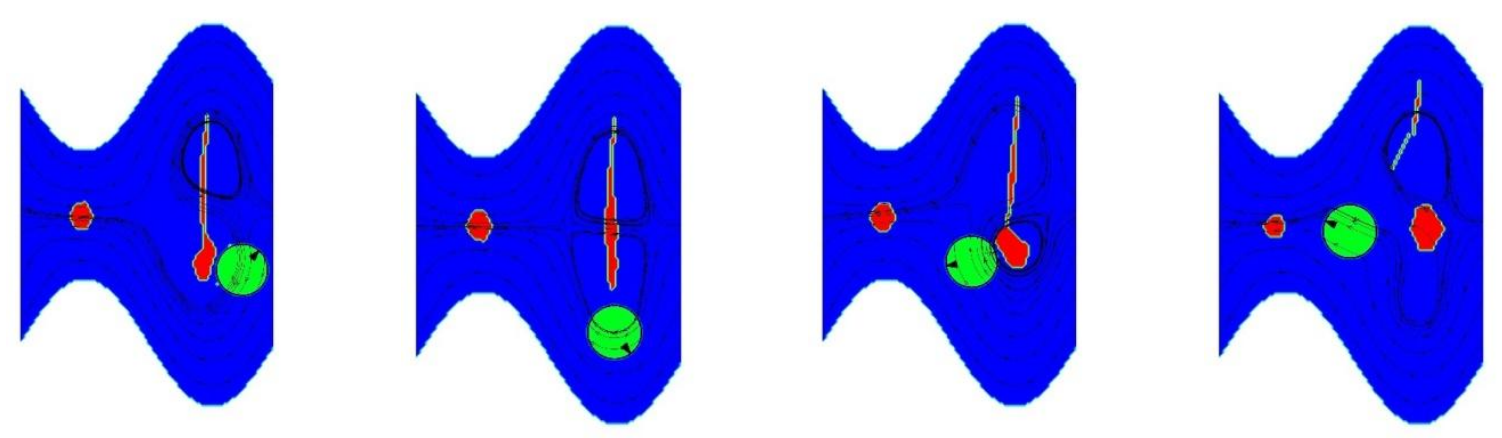

$$
\Lambda=23
$$

$$
\Lambda=24
$$
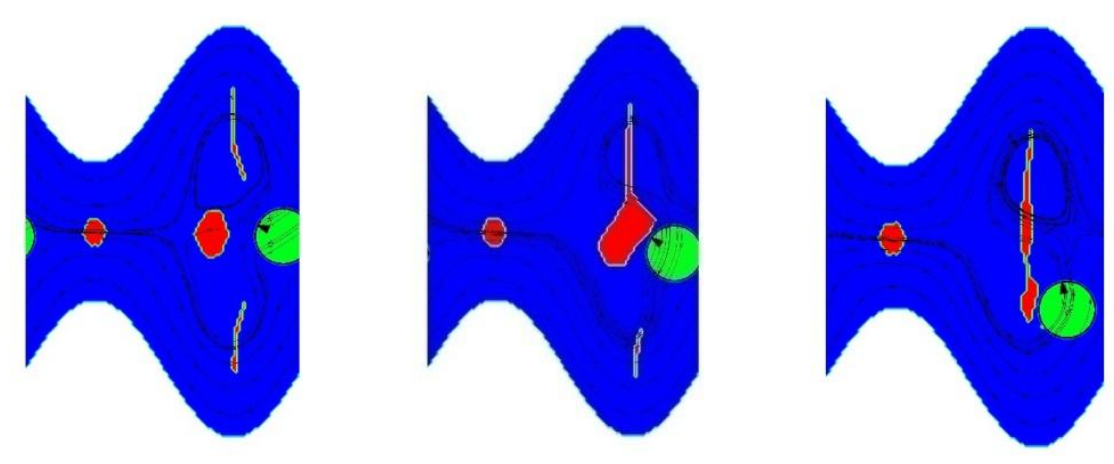

$\Lambda=27$

$$
\Lambda=28
$$

$$
\Lambda=29
$$

$$
\Lambda=30
$$
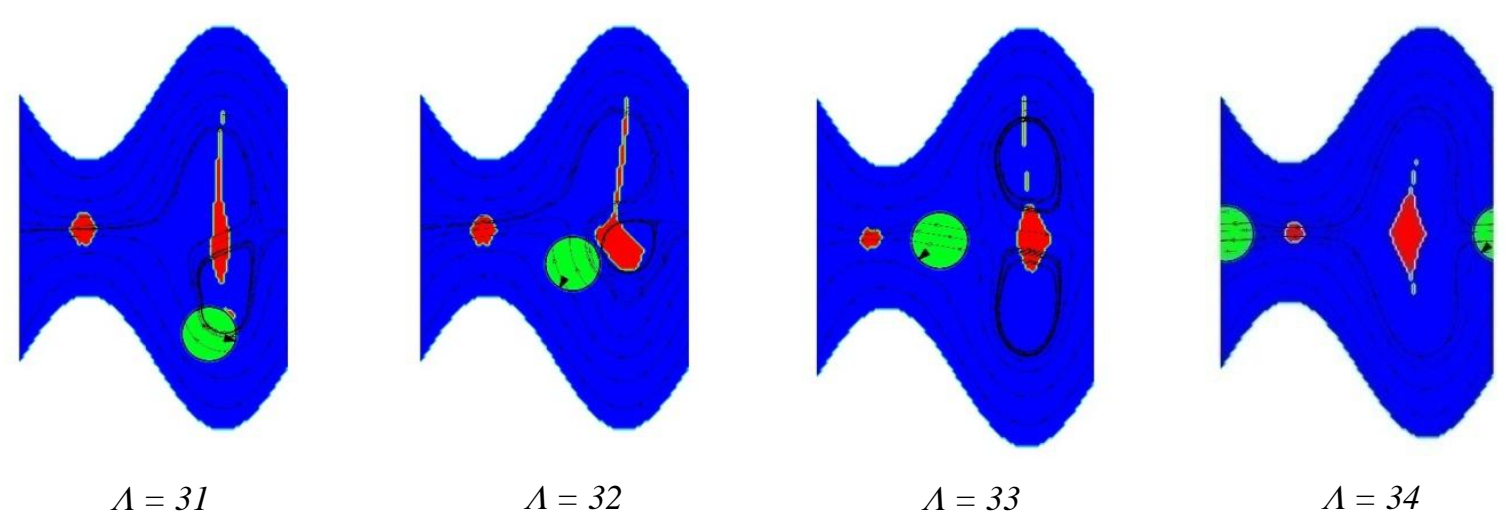

$\Lambda=31$

$\Lambda=32$

Figure 17 


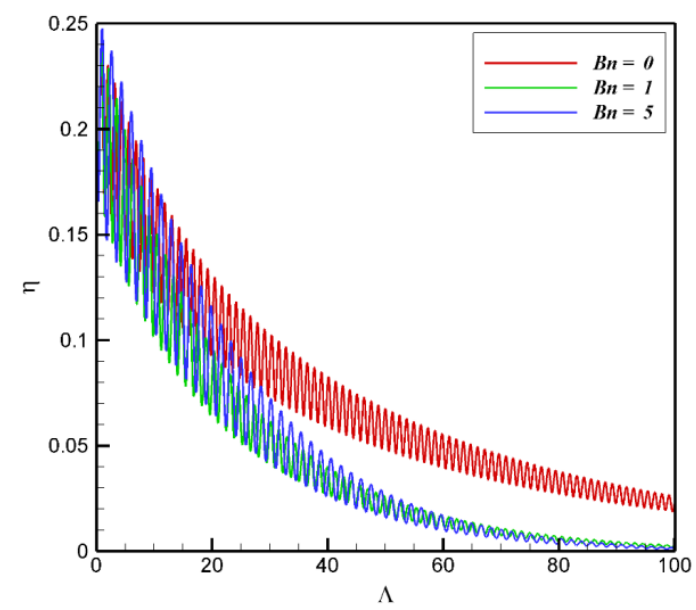

(a)

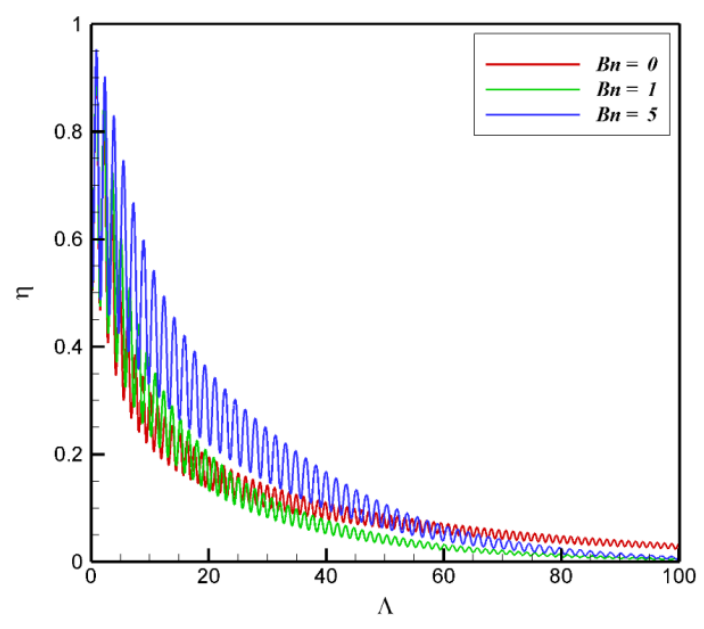

(b)

Figure 18

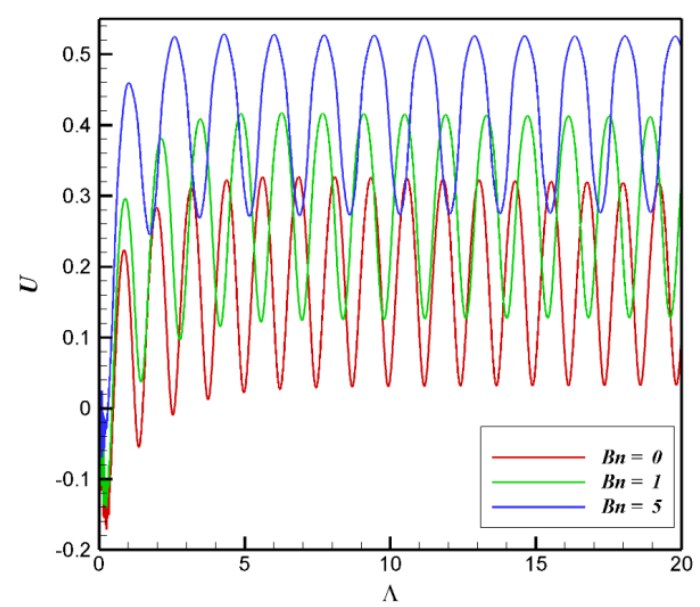

(a)

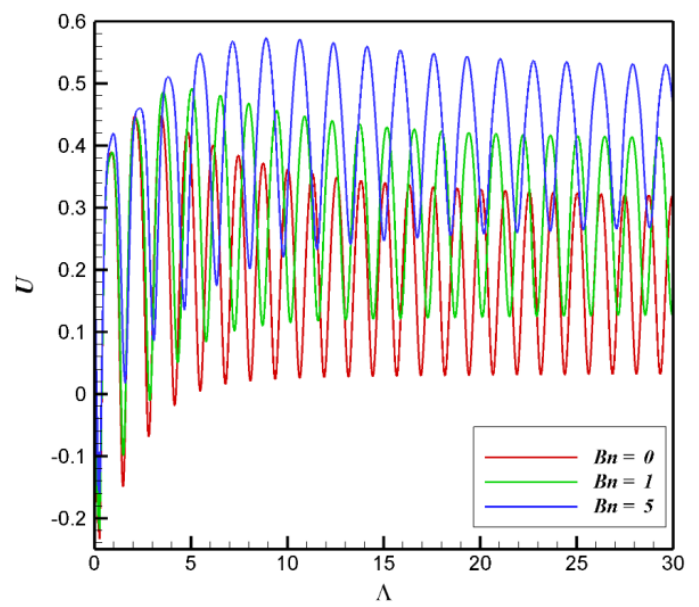

(b)

Figure 19 


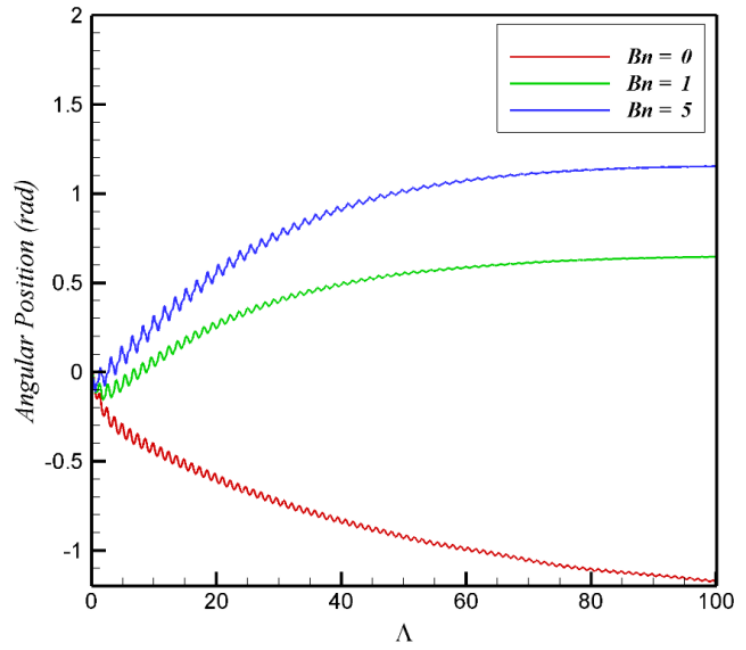

(a)

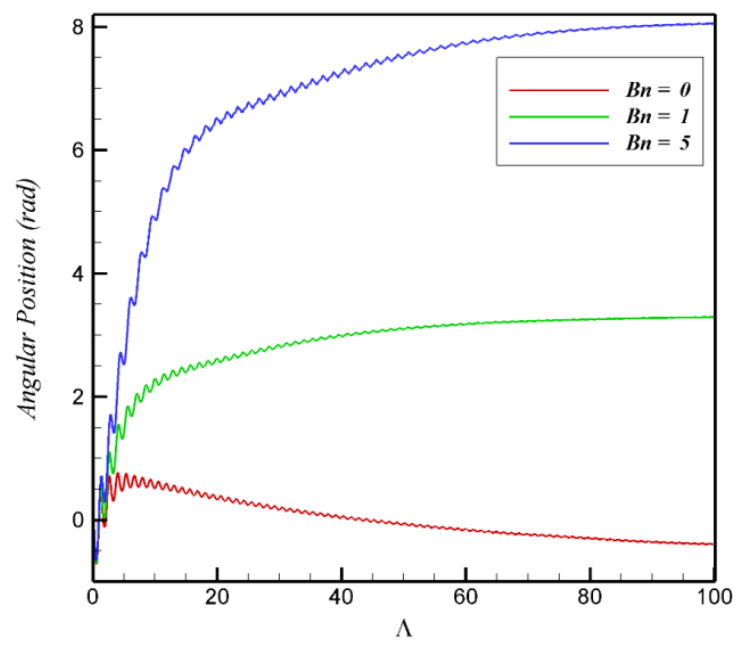

(b)

Figure 20

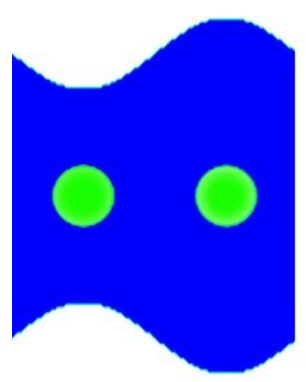

Case A

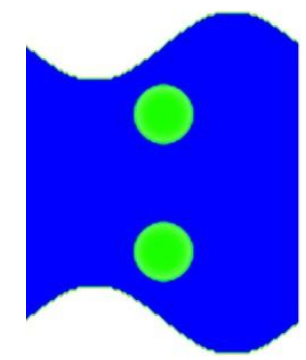

Case B

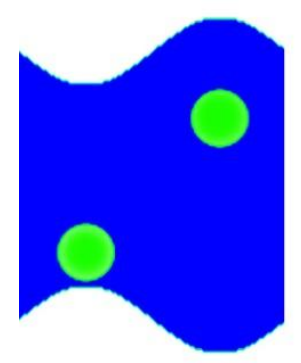

Case C

Figure 21 


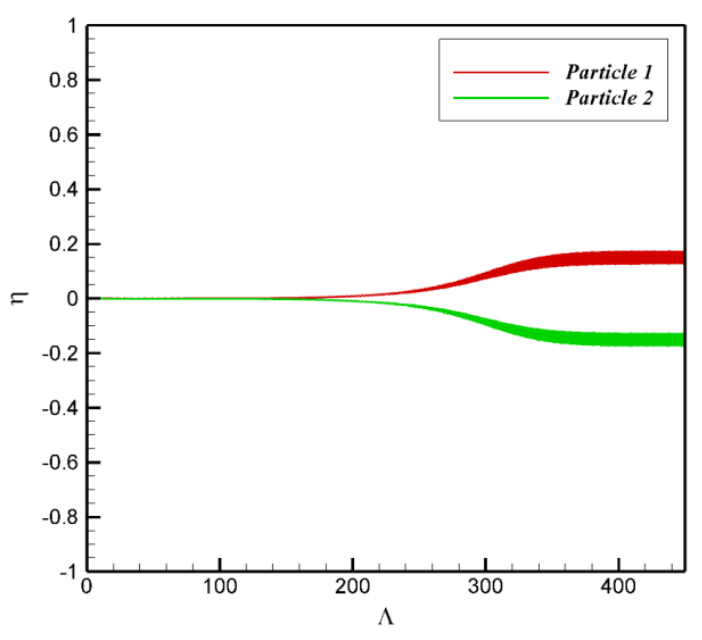

(a)

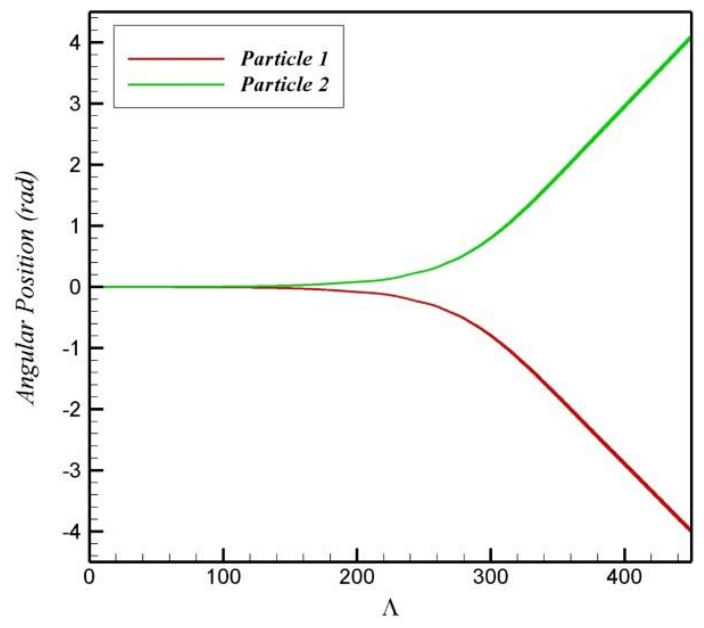

(b)

Figure 22

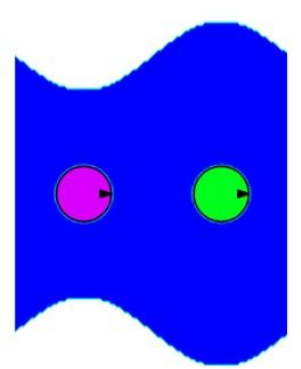

$\Lambda=0$

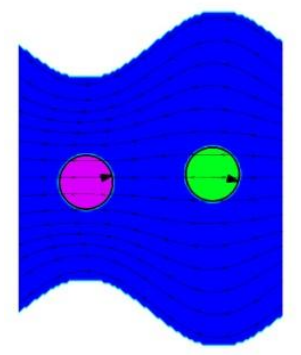

$\Lambda=250$

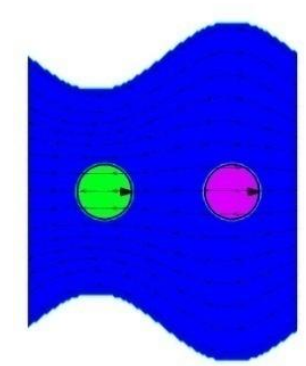

$\Lambda=50$

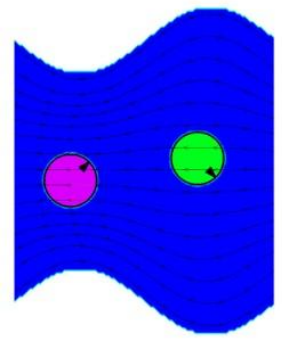

$\Lambda=300$

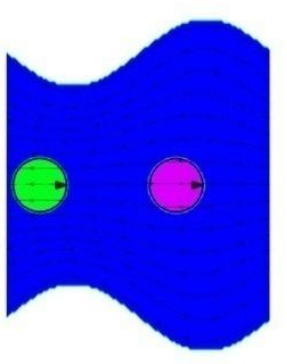

$\Lambda=100$

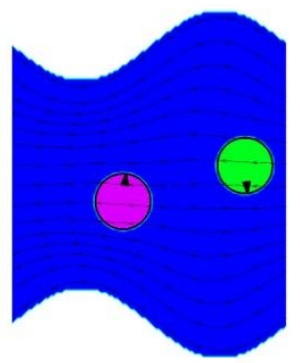

$\Lambda=335$

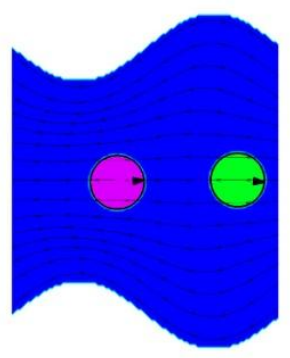

$\Lambda=200$

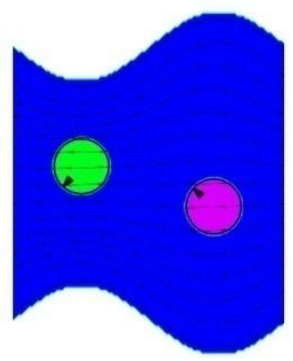

$\Lambda=375$

Figure 23 


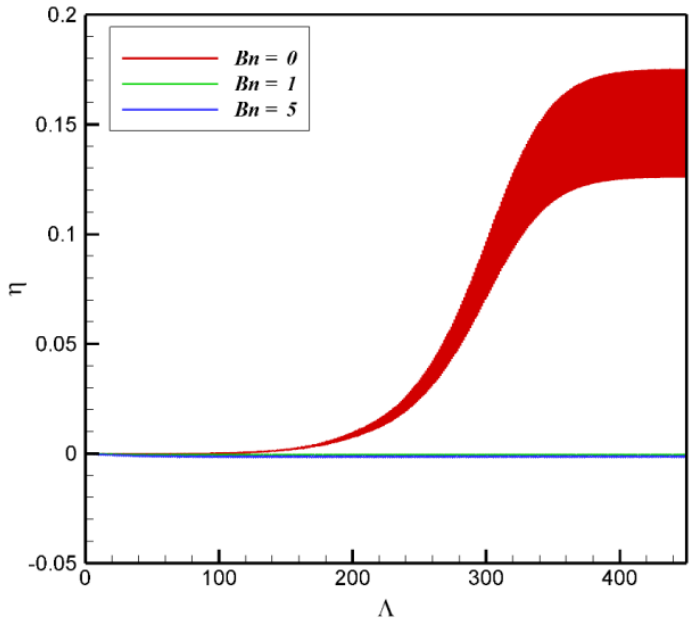

(a)

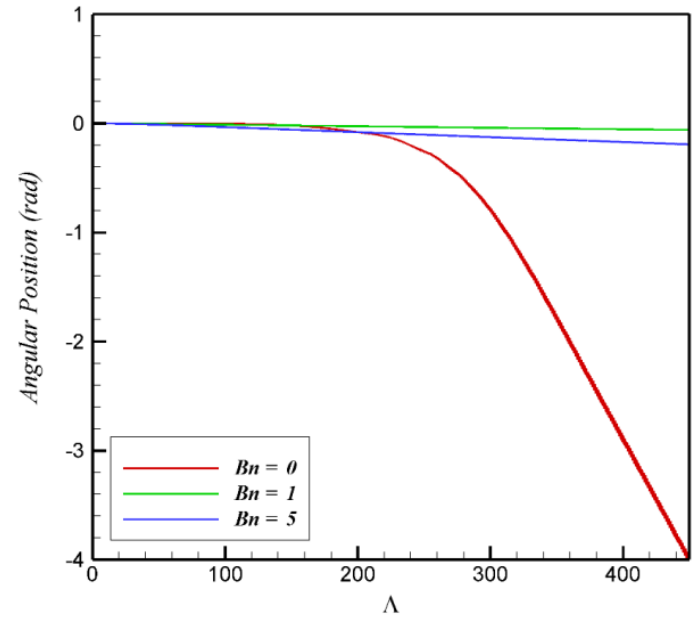

(b)

Figure 24

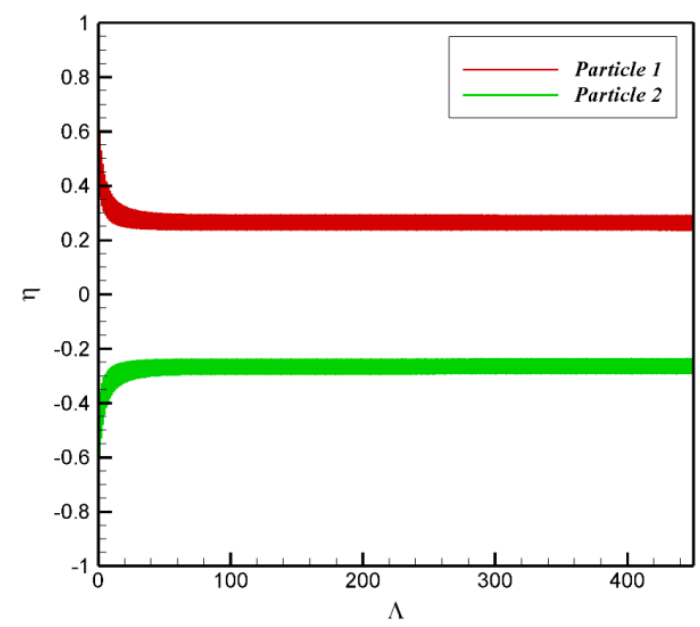

(a)

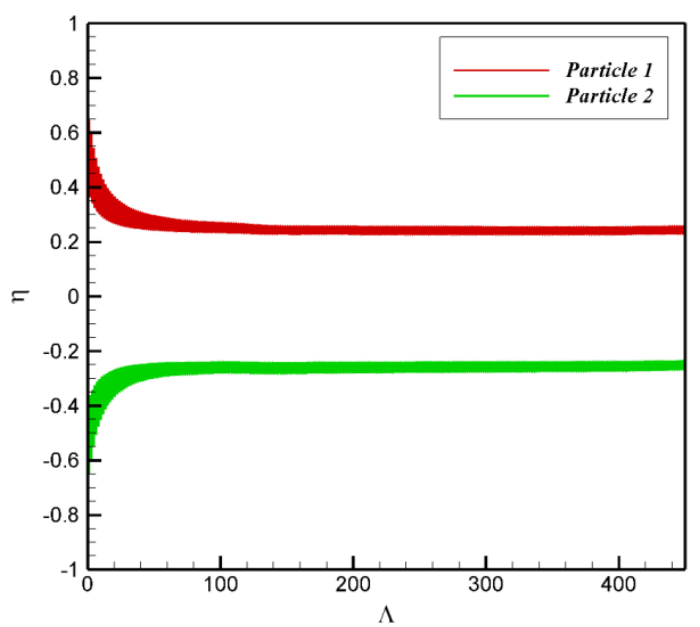

(b)

Figure 25 


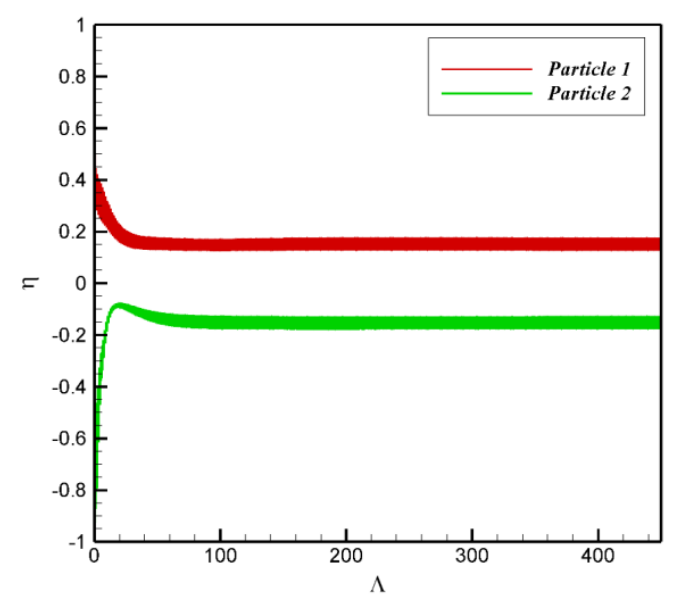

(a)

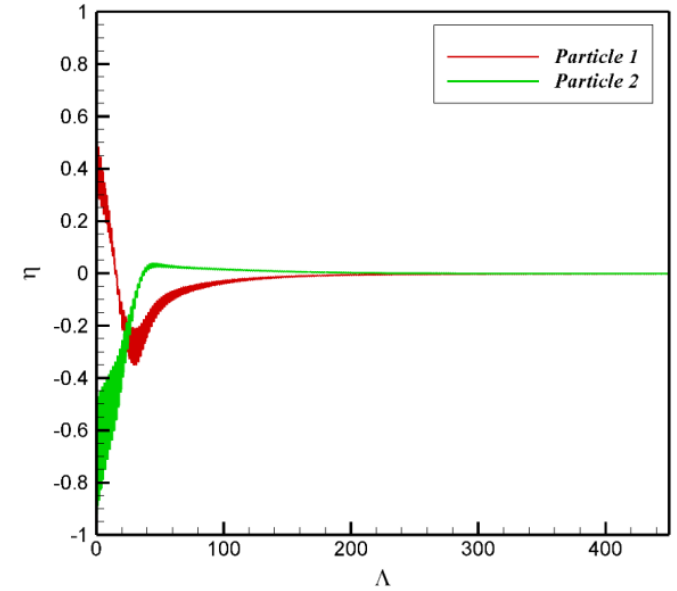

(b)

Figure 26

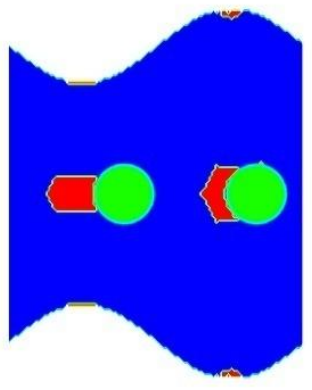

Case A

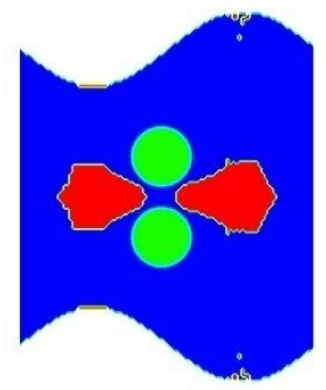

Case B

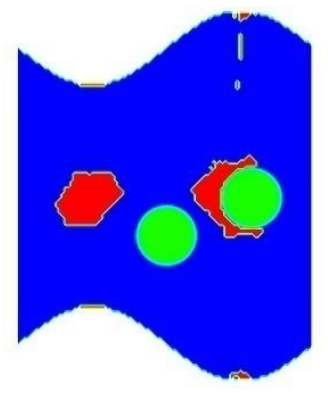

Case C

Figure 27 


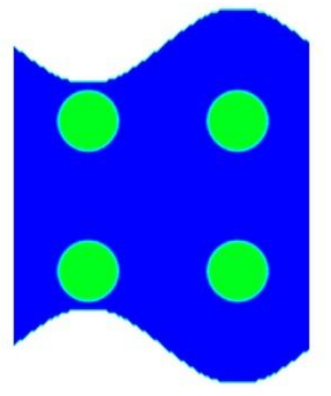

Case A

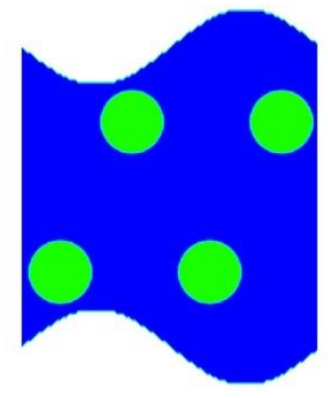

Case B

Figure 28

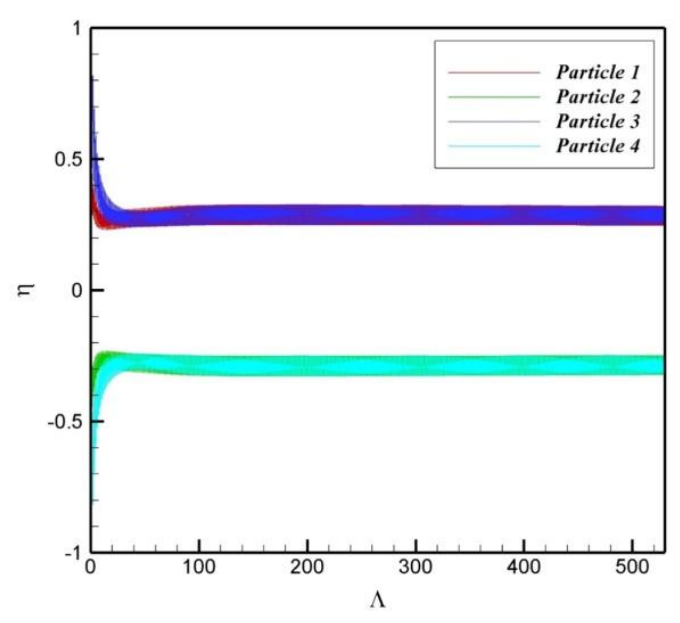

$\mathrm{Bn}=0$

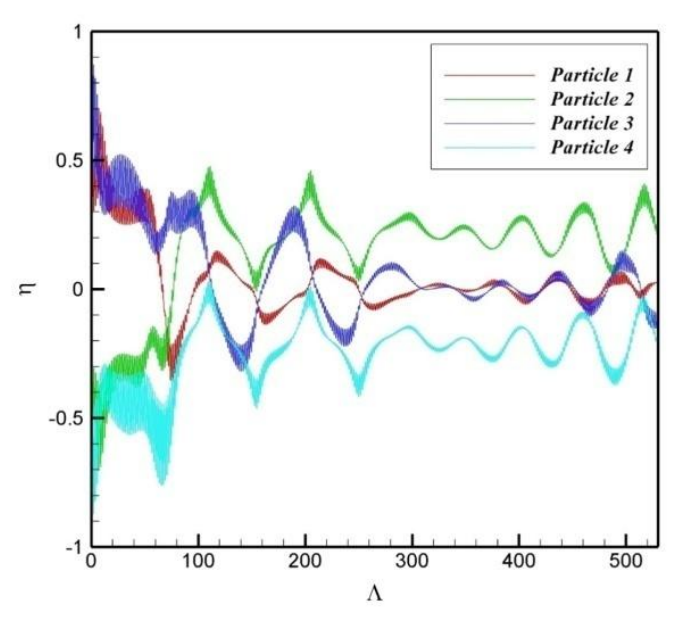

$\mathrm{Bn}=5$

Figure 29 


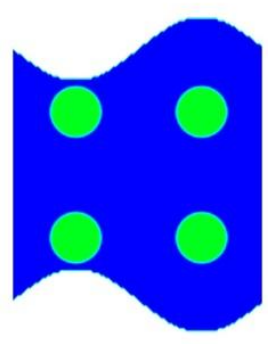

$\Lambda=0$

$\Lambda=40$

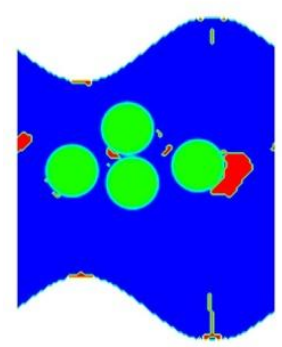

$\Lambda=110$

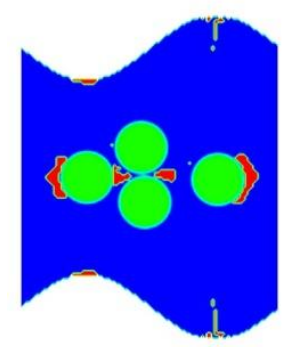

$\Lambda=345$

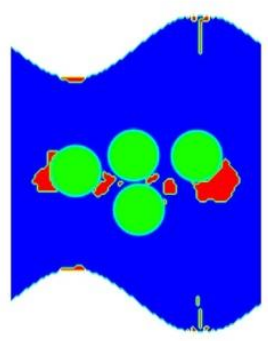

$\Lambda=500$

Figure 30

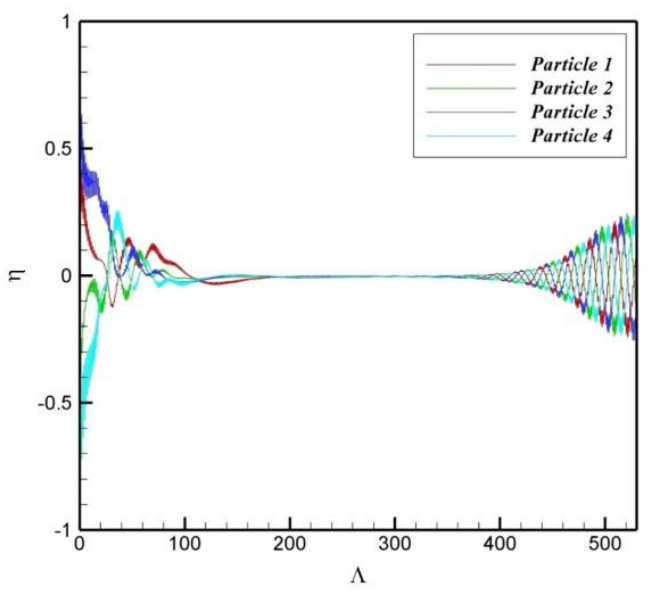

$\mathrm{Bn}=0$

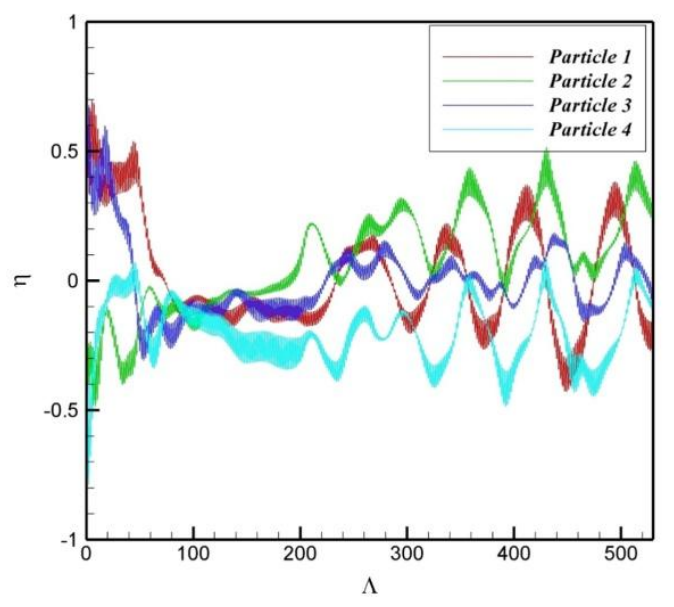

$\mathrm{Bn}=5$

Figure 31 

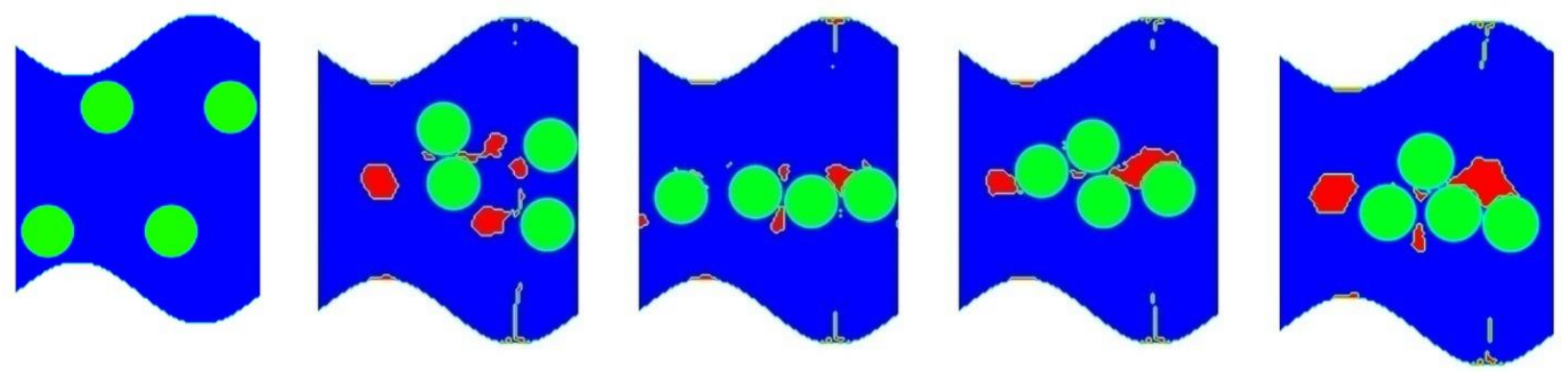

$$
\Lambda=0
$$

$$
\Lambda=30
$$

$\Lambda=115$

$\Lambda=290$

$\Lambda=540$

Figure 32 